\title{
Small Area Estimation under a Multivariate Linear Model for Repeated measures Data
}

\author{
Innocent Ngaruye, Joseph Nzabanita, Dietrich von Rosen and Martin Singull \\ Journal Article
}

\section{Tweet}

N.B.: When citing this work, cite the original article.

This is an electronic version of an article published in:

Innocent Ngaruye, Joseph Nzabanita, Dietrich von Rosen and Martin Singull, Small Area Estimation under a Multivariate Linear Model for Repeated measures Data, Communications in Statistics - Theory and Methods, 2016

Communications in Statistics - Theory and Methods is available online at informaworldTM: http://dx.doi.org/10.1080/03610926.2016.1248784

Copyright: Taylor \& Francis: STM, Behavioural Science and Public Health Titles

http://www.tandf.co.uk/journals/default.asp

Postprint available at: Linköping University Electronic Press

http://urn.kb.se/resolve?urn=urn:nbn:se:liu:diva-137116

I..0 


\title{
Small Area Estimation under a Multivariate Linear Model for Repeated measures Data
}

\author{
Innocent Ngaruye ${ }^{* 1,2}$, Joseph Nzabanita ${ }^{2}$, Dietrich von Rosen ${ }^{1,3}$, and Martin \\ Singull ${ }^{1}$ \\ ${ }^{1}$ Department of Mathematics, Linköping University \\ ${ }^{2}$ Department of Mathematics, College of Science and Technology, University of Rwanda \\ ${ }^{3}$ Department of Energy and Technology, Swedish University of Agricultural Sciences
}

\begin{abstract}
In this article, Small Area Estimation under a Multivariate Linear model for repeated measures data is considered. The proposed model aims to get a model which borrows strength both across small areas and over time. The model accounts for repeated surveys, grouped response units and random effects variations. Estimation of model parameters is discussed within a likelihood based approach. Prediction of random effects, small area means across time points and per group units are derived. A parametric bootstrap method is proposed for estimating the mean-squared errors of the predicted small area means. Results are supported by a simulation study.
\end{abstract}

Keywords: Maximum likelihood, Multivariate linear model, Prediction of random effects, Repeated measures data, Small Area Estimation

Mathematics Subject Classifications: 62F10, 62H12, 62D05

\section{Introduction}

Population surveys are carried out via sampling designs and data collection of individual units with intention of making statistical inferences about a larger population of which these units are members. These surveys are usually designed to provide efficient estimates of parameters of interest for large populations. In most cases, surveys are not originally designed to produce estimates for small domains and hence these domains are poorly represented in the sample. Thus, the surveys often provide very little information on a small area level and direct survey estimates on a target small area are not reliable due to a small sample size connected to this area.

*Address correspondence to Innocent Ngaruye, Department of Mathematics, Linköping University, SE-581 83 Linköping, Sweden; E-mail: innocent.ngaruye@liu.se 
In recent years, small area estimation methods have received much attention due to their usefulness in both public and private sectors and their demand has greatly increased worldwide. Several approaches and new developments in small area estimation have been investigated by different authors, for example, see Pfeffermann (2002, 2013); Rao (2003); Chambers and Clark (2012). One may also refer to Ghosh and Rao (1994) and Rao (2003) for some examples and case studies in small area estimation.

Model-based methods in small area estimation largely use linear mixed models with a focus on cross-sectional data. Some studies dealing with small area estimation problems for longitudinal surveys have been discussed by various authors, for example Consortium (2004), Nissinen (2009) and Singh and Sisodia (2011) who have used a design-based approach for repeated measures data in small area estimation and have developed direct, synthetic and composite estimators for small area means for repeated measures data at a given time point when the population units contain non-overlapping groups. It has been shown that the multivariate approach for modelbased methods in small area estimation may achieve substantial improvement over the usual univariate approach (Datta et al. 1999). Multivariate models in small area estimation such as the multivariate extensions of the univariate Fay-Herriot model originally proposed by Fay and Herriot (1979) and nested linear regression model introduced by Battese et al. (1988) have been considered by some authors such as Fuller and Harter (1987), Ghosh et al. (1991), Datta et al. (1999), Benavent and Morales (2016) among others. Although multivariate models have been adopted in small area estimation, Generalized Multivariate Analysis of Variance (GMANOVA) models, for example see Kollo and von Rosen (2005, Chapter 4), commonly known as Growth Curve models useful to study the treatment mean and the treatment change over time have not yet been considered.

In this article, we propose a model which borrows strength across both small areas and over time by incorporating simultaneously the effects of areas and time correlation. This model accounts for repeated surveys, grouped response units and random effects variations. The model presents a lot of advantages; it allows to find out the small area means at each time point, per group units and for all time points. Even though, it seems to be a complicated model, it is fairly realistic and can be verified empirically with reasonable sample sizes. Another advantage of the considered model is the possibility to model the pattern of changes or mean growth profiles over time by providing the shape of the mean trend over time, how the group units differ in their trajectories over time and the assessment of the variables which are associated with the pattern of change over time. Explicit estimators are obtained which is of great advantage when for example simulating mean-square errors (MSE) via a parametric bootstrap approach.

The article is organized as follows. After the Introduction, the second section is devoted to the model formulation and notation used and Section 3 deals with estimation of model parameters. Section 4 presents the model decomposition while Section 5 discusses the prediction of random effects and prediction of target small area means. Section 6 deals with proposed parametric bootstrap method. We end up by a simulation study in Section 7 and general concluding remarks are presented in Section 8. 


\section{Model formulation}

We consider repeated measurements on the response variable of interest, $y$, taken at $p$ time points $t_{1}, \ldots, t_{p}$ from a finite population $U$ of size $N$ which is partitioned into $m$, disjoint subpopulations $U_{1}, \ldots, U_{m}$ called small areas of sizes $N_{i}, i=1, \ldots, m$ such that $\sum_{i=1}^{m} N_{i}=N$. We also assume that in every area, there are $k$ different groups of units of size $N_{i g}$ for group $g$ such that $\sum_{g=1}^{k} N_{i g}=N_{i}$ and $\sum_{i=1}^{m} \sum_{g=1}^{k} N_{i g}=N$, where $N_{i g}$ is the size of group $g$ within the $i$-th area. Suppose that a sample $s=s_{1}, \ldots, s_{m}$ is selected from the population, for example, using simple random sampling without replacement, where $s_{i}$ is the sample of size $n_{i}$ observed from area $i$ and in each sample all groups are represented. The sample $s_{i}$ can be split into $s_{i g}, g=1, \ldots, k$ with corresponding sample sizes $n_{i g}$ for the $k$ groups. In this article, it is assumed that the sample remains the same over time.

Let $\boldsymbol{y}_{i j}$ be the $p$-vector of measurements over time on the $j$-th unit, in the $i$-th small area which are supposed to stem from a survey study. That is $\boldsymbol{y}_{i j}=\left(y_{i j 1}, \ldots, y_{i j p}\right)^{\prime}, \quad j=$ $1, \ldots, n_{i}, \quad i=1, \ldots, m$.

We assume the mean growth of the $j$ th unit in area $i$ for each group to be a polynomial in time of degree $q-1$. Furthermore, we suppose that we have auxiliary data $\boldsymbol{x}_{i j}$ of $r$ concomitant variables (covariates) whose values are known for all units in all $m$ small areas. These auxiliary variables are included in the model to strengthen the limited sample sizes from areas and are supposed to be constant over time. The values $\boldsymbol{x}_{i j}$ can be the values of a past survey from the same area and/or the values of other variables that are related to the variable of interest. Moreover, they can include register based information or the data measured for characteristics of interest in other similar areas.

The relationship between $\boldsymbol{y}_{i j}$ and $\boldsymbol{x}_{i j}$ in each small area is not always considered to be as the same as the relationship between the variables in the population as whole (Chambers and Clark, 2012). Therefore, we add an area specific term to allow them to better account for the between area variability in the distribution of $\boldsymbol{y}_{i j}$. That is, we suppose $u_{i t}$ random area-effects which vary over time, assumed to be independent and identically distributed with mean zero and variance $\sigma_{u t}^{2}$. Thus, for each one of the $k$ groups, the unit level regression model for the $j$-th unit coming from the small area $i$ at time $t$ can be expressed by

$$
y_{i j t}=\beta_{0}+\beta_{1} t+\cdots+\beta_{q} t^{q-1}+\gamma^{\prime} \boldsymbol{x}_{i j}+u_{i t}+e_{i j t}, i=1, \ldots, m ; j=1, \ldots, N_{i} ; t=t_{1}, \ldots, t_{p},
$$

where $e_{i j t}$ are random errors assumed to be i.i.d normal with mean zero and variance $\sigma_{e}^{2}$ independent of $u_{i t}$. The $\gamma$ is a vector of fixed regression coefficients representing the effects of auxiliary variables. The $\beta_{0}, \ldots, \beta_{q}$ are unknown parameters assumed to be the same in all areas under the assumption that there is no area interaction effect with in time. 
For all time points, the model can be written in matrix form as

$$
\boldsymbol{y}_{i j}=\boldsymbol{A} \boldsymbol{\beta}+\mathbf{1}_{p} \boldsymbol{\gamma}^{\prime} \boldsymbol{x}_{i j}+\boldsymbol{u}_{i}+\boldsymbol{e}_{i j}, \quad \boldsymbol{A}=\left(\begin{array}{ccccc}
1 & t_{1} & t_{1}^{2} & \cdots & t_{1}^{q-1} \\
1 & t_{2} & t_{2}^{2} & \cdots & t_{2}^{q-1} \\
\vdots & \vdots & \vdots & & \vdots \\
1 & t_{p} & t_{p}^{2} & \cdots & t_{p}^{q-1}
\end{array}\right)
$$

and $\mathbf{1}_{p}$ is a $p$-vector of ones. The vector $\boldsymbol{u}_{i}$ is assumed to be multivariate normally distributed with zero mean and unknown covariance matrix $\boldsymbol{\Sigma}_{u}$. Collecting the vectors $\boldsymbol{y}_{i j}$ for all units in small area $i$ coming from the group $g$ gives

$$
\boldsymbol{Y}_{i g}=\boldsymbol{A} \boldsymbol{\beta}_{g} \mathbf{1}_{N_{i g}}^{\prime}+\mathbf{1}_{p} \boldsymbol{\gamma}^{\prime} \boldsymbol{X}_{i g}+\boldsymbol{u}_{i} \boldsymbol{z}_{i g}^{\prime}+\boldsymbol{E}_{i g}, i=1, \ldots, m, g=1, \ldots, k,
$$

where $\boldsymbol{Y}_{i g}=\left(\boldsymbol{y}_{i 1}, \cdots, \boldsymbol{y}_{i N_{i g}}\right) ; \quad \boldsymbol{X}_{i g}=\left(\boldsymbol{x}_{i 1}, \cdots, \boldsymbol{x}_{i N_{i g}}\right) ; \quad \boldsymbol{z}_{i g}=\frac{1}{\sqrt{N_{i g}}} \mathbf{1}_{N_{i g}}$ and $\quad \boldsymbol{E}_{i g}=$ $\left(\boldsymbol{e}_{i 1}, \cdots, \boldsymbol{e}_{i N_{i g}}\right)$.

Since we are interested in every group unit, we need to include a known design matrix $\boldsymbol{C}_{i}: k \times N_{i}$ of group separation indicators when collecting all $k$ groups in the $i$-th area, which are supposed to be the same in all areas. The model at small area level for the $k$ groups is then written as

$$
\boldsymbol{Y}_{i}=\boldsymbol{A} \boldsymbol{B} \boldsymbol{C}_{i}+\mathbf{1}_{p} \boldsymbol{\gamma}^{\prime} \boldsymbol{X}_{i}+\boldsymbol{u}_{i} \boldsymbol{z}_{i}^{\prime}+\boldsymbol{E}_{i}, \quad i=1, \ldots, m,
$$

where $\boldsymbol{Y}_{i}=\left(\boldsymbol{Y}_{i 1}, \cdots, \boldsymbol{Y}_{i k}\right) ; \quad \boldsymbol{B}=\left(\boldsymbol{\beta}_{1}, \cdots, \boldsymbol{\beta}_{k}\right): q \times k ; \quad \boldsymbol{X}_{i}=\left(\boldsymbol{X}_{i 1}, \cdots, \boldsymbol{X}_{i k}\right) ; \quad \boldsymbol{z}_{i}=$ $\frac{1}{\sqrt{N_{i}}} \mathbf{1}_{N_{i}} ; \quad \boldsymbol{E}_{i g}=\left(\boldsymbol{e}_{i 1}, \cdots, \boldsymbol{e}_{i k}\right) ;$ and $\quad \boldsymbol{C}_{i}=\left(\begin{array}{ccc}\mathbf{1}_{N_{i 1}}^{\prime} & & \mathbf{0} \\ & \ddots & \\ \mathbf{0} & & \mathbf{1}_{N_{i k}}^{\prime}\end{array}\right)$.

The error term $\boldsymbol{E}_{i}$ is matrix normally distributed with mean zero, covariance matrix between rows $\boldsymbol{\Sigma}_{e}=\sigma_{e}^{2} \boldsymbol{I}_{p}$ and independent columns, denoted by $\boldsymbol{E}_{i} \sim \mathcal{N}_{p, N_{i}}\left(\mathbf{0}, \boldsymbol{\Sigma}_{e}, \boldsymbol{I}_{N_{i}}\right)$. One may refer, for example, to Kollo and von Rosen (2005) about matrix normal distribution properties. It is natural to suppose $\sigma_{e}^{2}$ to be known since the error stems from the survey and is connected only to the sample sizes not to model assumptions of the mean. Thus $\sigma_{e}^{2}$ will not be estimated. The matrix $\boldsymbol{Y}_{i}$ is a $p \times N_{i}$ data matrix; $\boldsymbol{A}$ is a $p \times q, q \leq p$ known within individuals design matrix for fixed effects; $\boldsymbol{B}$ is $q \times k$ unknown parameter matrix; $\boldsymbol{C}_{i}$ with $\operatorname{rank}\left(\boldsymbol{C}_{i}\right)+p \leq N_{i}$ is a $k \times N_{i}$ known between individuals design matrix for fixed effects and $\boldsymbol{X}_{i}$ is a $r \times N_{i}$ known matrix taking the values of the covariates.

Combining all small areas for $N$ units, we obtain the following model which constitutes the basis for our technical treatment.

Definition 2.1. The multivariate linear regression model for repeated measurements over $p$ time points, combining all disjoint $m$ small areas and all $N$ units divided into $k$ non-overlapping group units is given by

$$
\boldsymbol{Y}=\boldsymbol{A B H C}+\mathbf{1}_{p} \boldsymbol{\gamma}^{\prime} \boldsymbol{X}+\boldsymbol{U} \boldsymbol{Z}+\boldsymbol{E},
$$


where

$$
\begin{aligned}
& \boldsymbol{Y}_{p \times N}=\left[\boldsymbol{Y}_{1}, \cdots, \boldsymbol{Y}_{m}\right] ; \quad \boldsymbol{H}_{k \times m k}=\left[\boldsymbol{I}_{k}: \cdots: \boldsymbol{I}_{k}\right] ; \boldsymbol{X}_{r \times N}=\left[\boldsymbol{X}_{1}, \cdots, \boldsymbol{X}_{m}\right] ; \\
& \boldsymbol{U}_{p \times m}=\left[\boldsymbol{u}_{1}, \cdots, \boldsymbol{u}_{m}\right] ; \quad \boldsymbol{E}=\left[\boldsymbol{E}_{1}, \cdots, \boldsymbol{E}_{m}\right] ; \quad \boldsymbol{C}_{m k \times N}=\left(\begin{array}{ccc}
\boldsymbol{C}_{1} & & \mathbf{0} \\
& \ddots & \\
\mathbf{0} & & \boldsymbol{C}_{m}
\end{array}\right), \\
& \boldsymbol{Z}_{m \times N}=\left(\begin{array}{ccc}
\boldsymbol{z}_{1}^{\prime} & & \mathbf{0} \\
& \ddots & \\
\mathbf{0} & & \boldsymbol{z}_{m}^{\prime}
\end{array}\right), \operatorname{rank}(\boldsymbol{C})+p \leq N,
\end{aligned}
$$

where $\boldsymbol{E} \sim \mathcal{N}_{p, N}\left(\mathbf{0}, \boldsymbol{\Sigma}_{e}, \boldsymbol{I}_{N}\right), \quad \boldsymbol{U} \sim \mathcal{N}_{p, m}\left(\mathbf{0}, \boldsymbol{\Sigma}_{u}, \boldsymbol{I}_{m}\right), \quad p \leq m, \boldsymbol{\Sigma}_{u}$ is an arbitrary positive definite matrix.

\section{Decomposition of the model into sub-models}

The model defined in (2) is considered as a random effects growth curve model with covariates, see for example Yokoyama and Fujikoshi (1992), Yokoyama (1995) and Nummi (1997). The estimation in this article is performed using a likelihood based approach.

To facilitate the estimation approach, we make some transformations. Moreover, in the following, we use the notation $\boldsymbol{A}^{\circ}$ for any matrix of full rank spanning $\mathcal{C}(\boldsymbol{A})^{\perp}$, i.e., $\mathcal{C}\left(\boldsymbol{A}^{o}\right)=$ $\mathcal{C}(\boldsymbol{A})^{\perp}$, where $\mathcal{C}(\boldsymbol{A})$ denotes the column vector space generated by the columns of the matrix $\boldsymbol{A}$. The matrix $\boldsymbol{A}^{-}$denotes an arbitrary generalized inverse of the matrix $\boldsymbol{A}$ such that $\boldsymbol{A} \boldsymbol{A}^{-} \boldsymbol{A}=\boldsymbol{A}$. We also denote by $\boldsymbol{P}_{\boldsymbol{A}}=\boldsymbol{A}\left(\boldsymbol{A}^{\prime} \boldsymbol{A}\right)^{-} \boldsymbol{A}^{\prime}$ and $\boldsymbol{Q}_{\boldsymbol{A}}=\boldsymbol{I}-\boldsymbol{P}_{\boldsymbol{A}}$ the orthogonal projection matrices onto the column space $\mathcal{C}(\boldsymbol{A})$ and onto its orthogonal complement $\mathcal{C}(\boldsymbol{A})^{\perp}$, respectively.

First of all, we observe that the matrices $\boldsymbol{Z}, \boldsymbol{H}, \boldsymbol{C}$ in model (2) are of full row rank and the matrix $\boldsymbol{A}$ is of full column rank. Moreover, $\mathcal{C}\left(\boldsymbol{Z}^{\prime}\right) \subseteq \mathcal{C}\left(\boldsymbol{C}^{\prime}\right)$ and $\boldsymbol{Z} \boldsymbol{Z}^{\prime}=\boldsymbol{I}_{m}$. These relations imply that $\left(\boldsymbol{C} \boldsymbol{C}^{\prime}\right)^{-1 / 2} \boldsymbol{C} \boldsymbol{Z}^{\prime} \boldsymbol{Z} \boldsymbol{C}^{\prime}\left(\boldsymbol{C} \boldsymbol{C}^{\prime}\right)^{-1 / 2}$ is an idempotent matrix and thus can be diagonalized by an orthogonal matrix, say $\boldsymbol{\Gamma}$, with corresponding eigenvalues 1 and 0 , i.e.,

$$
\left(\boldsymbol{C} \boldsymbol{C}^{\prime}\right)^{-1 / 2} \boldsymbol{C} \boldsymbol{Z}^{\prime} \boldsymbol{Z} \boldsymbol{C}^{\prime}\left(\boldsymbol{C} \boldsymbol{C}^{\prime}\right)^{-1 / 2}=\boldsymbol{\Gamma} \boldsymbol{D} \boldsymbol{\Gamma}^{\prime}=\boldsymbol{\Gamma}\left(\begin{array}{cc}
\boldsymbol{I}_{m} & \mathbf{0} \\
\mathbf{0} & \mathbf{0}
\end{array}\right) \boldsymbol{\Gamma}^{\prime} .
$$

Partition $\boldsymbol{\Gamma}=\left[\boldsymbol{\Gamma}_{1}: \boldsymbol{\Gamma}_{2}\right]$ such that $\boldsymbol{\Gamma}_{1}$ corresponds to the block $\boldsymbol{I}_{m}$ and $\boldsymbol{\Gamma}_{2}$ corresponds to the block $\mathbf{0}$, with $\boldsymbol{\Gamma}_{1}: m k \times m$ and $\boldsymbol{\Gamma}_{2}: m k \times(m k-m)$. It follows that $\boldsymbol{\Gamma}_{1}^{\prime} \boldsymbol{\Gamma}_{1}=\boldsymbol{I}_{m}$ and $\boldsymbol{\Gamma}_{2}^{\prime} \boldsymbol{\Gamma}_{2}=\boldsymbol{I}_{m k-m}$

Choose $\boldsymbol{C}^{\prime o}$ to be a matrix which columns are eigenvectors corresponding to the non zero eigenvalues of $\boldsymbol{Q}_{\boldsymbol{C}^{\prime}}$. Then we can apply a one to one transformation on 2 to get $[\boldsymbol{V}: \boldsymbol{W}]=$ 
$\boldsymbol{Y}\left[\boldsymbol{C}^{\prime}\left(\boldsymbol{C} \boldsymbol{C}^{\prime}\right)^{-1 / 2}: \boldsymbol{C}^{\prime o}\right]$, where

$$
\begin{aligned}
\boldsymbol{V} & =\boldsymbol{Y} \boldsymbol{C}^{\prime}\left(\boldsymbol{C} \boldsymbol{C}^{\prime}\right)^{-1 / 2} \\
& =\boldsymbol{A} \boldsymbol{B} \boldsymbol{H}\left(\boldsymbol{C} \boldsymbol{C}^{\prime}\right)^{1 / 2}+\mathbf{1}_{p} \boldsymbol{\gamma}^{\prime} \boldsymbol{X} \boldsymbol{C}^{\prime}\left(\boldsymbol{C} \boldsymbol{C}^{\prime}\right)^{-1 / 2}+(\boldsymbol{U} \boldsymbol{Z}+\boldsymbol{E}) \boldsymbol{C}^{\prime}\left(\boldsymbol{C} \boldsymbol{C}^{\prime}\right)^{-1 / 2}, \\
\boldsymbol{W} & =\boldsymbol{Y} \boldsymbol{C}^{\prime o}=\mathbf{1}_{p} \boldsymbol{\gamma}^{\prime} \boldsymbol{X} \boldsymbol{C}^{\prime o}+\boldsymbol{E} \boldsymbol{C}^{\prime o}
\end{aligned}
$$

since $\mathcal{C}\left(\boldsymbol{Z}^{\prime}\right) \subseteq \mathcal{C}\left(\boldsymbol{C}^{\prime}\right)$ implies that $\boldsymbol{Z} \boldsymbol{C}^{\prime}=\mathbf{0}$. Make a further transformation on model equation (3) so that $\left[\boldsymbol{V}_{1}: \boldsymbol{V}_{2}\right]=\boldsymbol{Y} \boldsymbol{C}^{\prime}\left(\boldsymbol{C} \boldsymbol{C}^{\prime}\right)^{-1 / 2}\left[\boldsymbol{\Gamma}_{1}: \boldsymbol{\Gamma}_{2}\right]$, where

$$
\begin{aligned}
\boldsymbol{V}_{1} & =\boldsymbol{Y} \boldsymbol{C}^{\prime}\left(\boldsymbol{C} \boldsymbol{C}^{\prime}\right)^{-1 / 2} \boldsymbol{\Gamma}_{1} \\
& =\boldsymbol{A} \boldsymbol{B} \boldsymbol{H}\left(\boldsymbol{C} \boldsymbol{C}^{\prime}\right)^{1 / 2} \boldsymbol{\Gamma}_{1}+\mathbf{1}_{p} \boldsymbol{\gamma}^{\prime} \boldsymbol{X} \boldsymbol{C}^{\prime}\left(\boldsymbol{C} \boldsymbol{C}^{\prime}\right)^{-1 / 2} \boldsymbol{\Gamma}_{1}+(\boldsymbol{U} \boldsymbol{Z}+\boldsymbol{E}) \boldsymbol{C}^{\prime}\left(\boldsymbol{C} \boldsymbol{C}^{\prime}\right)^{-1 / 2} \boldsymbol{\Gamma}_{1}, \\
\boldsymbol{V}_{2} & =\boldsymbol{Y} \boldsymbol{C}^{\prime}\left(\boldsymbol{C} \boldsymbol{C}^{\prime}\right)^{-1 / 2} \boldsymbol{\Gamma}_{2} \\
& =\boldsymbol{A} \boldsymbol{B} \boldsymbol{H}\left(\boldsymbol{C} \boldsymbol{C}^{\prime}\right)^{1 / 2} \boldsymbol{\Gamma}_{2}+\mathbf{1}_{p} \boldsymbol{\gamma}^{\prime} \boldsymbol{X} \boldsymbol{C}^{\prime}\left(\boldsymbol{C} \boldsymbol{C}^{\prime}\right)^{-1 / 2} \boldsymbol{\Gamma}_{2}+\boldsymbol{E} \boldsymbol{C}^{\prime}\left(\boldsymbol{C} \boldsymbol{C}^{\prime}\right)^{-1 / 2} \boldsymbol{\Gamma}_{2} .
\end{aligned}
$$

Observe that the above decomposition is important. It allows us to achieve matrix normally distributed sub-models (4), (5) and (6) from model (2). Typically for a matrix normally distributed variable is that its dispersion matrix consists of a Kronecker product of two interpretable dispersion matrices. The following Theorem 3.1 summarizes the above calculations.

Theorem 3.1. Let $\boldsymbol{V}_{1}, \boldsymbol{V}_{2}$ and $\boldsymbol{W}$ be as defined in (5), (6) and (4), respectively. Then,

$$
\begin{aligned}
& \boldsymbol{V}_{1} \sim \mathcal{N}_{p, m}\left(\boldsymbol{M}_{1}, \boldsymbol{\Sigma}_{u}+\boldsymbol{\Sigma}_{e}, \boldsymbol{I}_{m}\right), \\
& \boldsymbol{V}_{2} \sim \mathcal{N}_{p, m k-m}\left(\boldsymbol{M}_{2}, \boldsymbol{\Sigma}_{e}, \boldsymbol{I}_{m k-m}\right), \\
& \boldsymbol{W} \sim \mathcal{N}_{p, N-m k}\left(\boldsymbol{M}_{3}, \boldsymbol{\Sigma}_{e}, \boldsymbol{I}_{N-m k}\right), \\
& \text { with }\left[\boldsymbol{M}_{1}: \boldsymbol{M}_{2}\right]=\boldsymbol{A} \boldsymbol{B} \boldsymbol{H}\left(\boldsymbol{C} \boldsymbol{C}^{\prime}\right)^{1 / 2}+\mathbf{1}_{p} \boldsymbol{\gamma}^{\prime} \boldsymbol{X} \boldsymbol{C}^{\prime}\left(\boldsymbol{C} \boldsymbol{C}^{\prime}\right)^{-1 / 2}\left[\boldsymbol{\Gamma}_{1}: \boldsymbol{\Gamma}_{2}\right], \quad \boldsymbol{M}_{3}=\mathbf{1}_{p} \boldsymbol{\gamma}^{\prime} \boldsymbol{X} \boldsymbol{C}^{\prime o} .
\end{aligned}
$$

Furthermore, the matrices $\boldsymbol{V}_{1}, \boldsymbol{V}_{2}$ and $\boldsymbol{W}$ are independently distributed.

Proof. The independence of $\boldsymbol{V}_{1}, \boldsymbol{V}_{2}$ and $\boldsymbol{W}$ follows by the fact that the matrices $\boldsymbol{\Gamma}_{1}, \boldsymbol{\Gamma}_{2}$ and $\boldsymbol{C}^{\prime o}$ are pairwise orthogonal. Furthermore, since

$$
\begin{aligned}
& \boldsymbol{\Gamma}_{1}^{\prime}\left(\boldsymbol{C} \boldsymbol{C}^{\prime}\right)^{-1 / 2} \boldsymbol{C} \boldsymbol{Z}^{\prime} \boldsymbol{Z} \boldsymbol{C}^{\prime}\left(\boldsymbol{C} \boldsymbol{C}^{\prime}\right)^{-1 / 2} \boldsymbol{\Gamma}_{1}=\boldsymbol{\Gamma}_{1}^{\prime} \boldsymbol{\Gamma} \boldsymbol{D} \boldsymbol{\Gamma}^{\prime} \boldsymbol{\Gamma}_{1}=\boldsymbol{I}_{m}, \\
& \boldsymbol{\Gamma}_{1}^{\prime}\left(\boldsymbol{C} \boldsymbol{C}^{\prime}\right)^{-1 / 2} \boldsymbol{C} \boldsymbol{C}^{\prime}\left(\boldsymbol{C} \boldsymbol{C}^{\prime}\right)^{-1 / 2} \boldsymbol{\Gamma}_{1}=\boldsymbol{I}_{m}, \\
& \boldsymbol{\Gamma}_{2}^{\prime}\left(\boldsymbol{C} \boldsymbol{C}^{\prime}\right)^{-1 / 2} \boldsymbol{C} \boldsymbol{C}^{\prime}\left(\boldsymbol{C} \boldsymbol{C}^{\prime}\right)^{-1 / 2} \boldsymbol{\Gamma}_{2}=\boldsymbol{I}_{m k-m}, \quad\left(\boldsymbol{C}^{\prime o}\right)^{\prime} \boldsymbol{C}^{\prime o}=\boldsymbol{I}_{N-m k} .
\end{aligned}
$$

Hence,

$$
\begin{aligned}
& (\boldsymbol{U} \boldsymbol{Z}+\boldsymbol{E}) \boldsymbol{C}^{\prime}\left(\boldsymbol{C} \boldsymbol{C}^{\prime}\right)^{-1 / 2} \boldsymbol{\Gamma}_{1} \sim \mathcal{N}_{p, m}\left(\mathbf{0}, \boldsymbol{\Sigma}_{u}+\boldsymbol{\Sigma}_{e}, \boldsymbol{I}_{m}\right) \\
& \boldsymbol{E} \boldsymbol{C}^{\prime}\left(\boldsymbol{C} \boldsymbol{C}^{\prime}\right)^{-1 / 2} \boldsymbol{\Gamma}_{2} \sim \mathcal{N}_{p, m k-m}\left(\mathbf{0}, \boldsymbol{\Sigma}_{e}, \boldsymbol{I}_{m k-m}\right) \\
& \boldsymbol{E} \boldsymbol{C}^{\prime o} \sim \mathcal{N}_{p, N-m k}\left(\mathbf{0}, \boldsymbol{\Sigma}_{e}, \boldsymbol{I}_{N-m k}\right), \text { which completes the proof. }
\end{aligned}
$$




\section{Estimation of the mean and covariance}

We assume that the model defined in (2) holds for both sampled and nonsampled population units, i.e., the sampling method is non-informative. For the purpose of estimation, in this section we consider the corresponding model for the sampled data. Consider the three models $\boldsymbol{W}, \boldsymbol{V}_{1}$ and $\boldsymbol{V}_{2}$ given in (4), (5) and (6), respectively and $\boldsymbol{K}_{1}=\boldsymbol{H}\left(\boldsymbol{C} \boldsymbol{C}^{\prime}\right)^{1 / 2} \boldsymbol{\Gamma}_{1}, \quad \boldsymbol{K}_{2}=$ $\boldsymbol{H}\left(\boldsymbol{C} \boldsymbol{C}^{\prime}\right)^{1 / 2} \boldsymbol{\Gamma}_{2}, \quad \boldsymbol{R}_{1}=\boldsymbol{C}^{\prime}\left(\boldsymbol{C} \boldsymbol{C}^{\prime}\right)^{-1 / 2} \boldsymbol{\Gamma}_{1}, \quad \boldsymbol{R}_{2}=\boldsymbol{C}^{\prime}\left(\boldsymbol{C} \boldsymbol{C}^{\prime}\right)^{-1 / 2} \boldsymbol{\Gamma}_{2}$. Then,

$$
\begin{aligned}
& \boldsymbol{W}=\boldsymbol{Y} \boldsymbol{C}^{\prime o}=\mathbf{1}_{p} \boldsymbol{\gamma}^{\prime} \boldsymbol{X} \boldsymbol{C}^{\prime o}+\boldsymbol{E} \boldsymbol{C}^{\prime o}, \quad \boldsymbol{V}_{1}=\boldsymbol{Y} \boldsymbol{R}_{1}=\boldsymbol{A} \boldsymbol{B} \boldsymbol{K}_{1}+\mathbf{1}_{p} \boldsymbol{\gamma}^{\prime} \boldsymbol{X} \boldsymbol{R}_{1}+\boldsymbol{E} \boldsymbol{R}_{1}, \\
& \boldsymbol{V}_{2}=\boldsymbol{Y} \boldsymbol{R}_{2}=\boldsymbol{A} \boldsymbol{B} \boldsymbol{K}_{2}+\mathbf{1}_{p} \boldsymbol{\gamma}^{\prime} \boldsymbol{X} \boldsymbol{R}_{2}+\boldsymbol{E} \boldsymbol{R}_{2} .
\end{aligned}
$$

The corresponding log-likelihood of the joint density function of $\boldsymbol{W}$ and $\boldsymbol{V}_{2}$ is given by $l(\boldsymbol{\gamma}, \boldsymbol{B})=$ $l_{\boldsymbol{W}}(\gamma)+l_{\boldsymbol{V}_{2}}(\boldsymbol{\gamma}, \boldsymbol{B})$, where $l_{\boldsymbol{W}}(\gamma)$ and $l_{\boldsymbol{V}_{2}}(\boldsymbol{\gamma}, \boldsymbol{B})$ denote the log-likelihood functions for $\boldsymbol{W}$ and $\boldsymbol{V}_{2}$, respectively.

Differentiating the $\log$-likelihood with respect to $\gamma$ and $\boldsymbol{B}$ leads to the likelihood equations

$$
\begin{array}{r}
\left(\boldsymbol{X} \boldsymbol{C}^{\prime o}\left(\boldsymbol{C}^{\prime o}\right)^{\prime}+\boldsymbol{X} \boldsymbol{R}_{2} \boldsymbol{R}_{2}^{\prime}\right) \boldsymbol{Y}^{\prime} \mathbf{1}_{p}-p \boldsymbol{X}\left(\boldsymbol{C}^{\prime o}\left(\boldsymbol{C}^{\prime o}\right)^{\prime}+\boldsymbol{R}_{2} \boldsymbol{R}_{2}^{\prime}\right) \boldsymbol{X}^{\prime} \boldsymbol{\gamma}-\boldsymbol{X} \boldsymbol{R}_{2} \boldsymbol{K}_{2}^{\prime} \boldsymbol{B}^{\prime} \boldsymbol{A}^{\prime} \mathbf{1}_{p}=\mathbf{0} \\
\boldsymbol{A}^{\prime}\left(\boldsymbol{Y} \boldsymbol{R}_{2}-\boldsymbol{A} \boldsymbol{B} \boldsymbol{K}_{2}-\mathbf{1}_{p} \boldsymbol{\gamma}^{\prime} \boldsymbol{X} \boldsymbol{R}_{2}\right) \boldsymbol{K}_{2}^{\prime}=\mathbf{0}
\end{array}
$$

The proof of the next lemma can be found in the Appendix.

Lemma 4.1. The likelihood equation (7) admits a unique solution for the parameter vector $\boldsymbol{\gamma}$ if the matrix $\boldsymbol{X}$ is of full rank whereas the likelihood equation (8) admits a non-unique solution for the parameter matrix $\boldsymbol{B}$.

Equation (8) gives

$$
\boldsymbol{B}=\left(\boldsymbol{A}^{\prime} \boldsymbol{A}\right)^{-1} \boldsymbol{A}^{\prime} \boldsymbol{Y} \boldsymbol{R}_{2} \boldsymbol{K}_{2}^{\prime}\left(\boldsymbol{K}_{2} \boldsymbol{K}_{2}^{\prime}\right)^{-}-\left(\boldsymbol{A}^{\prime} \boldsymbol{A}\right)^{-1} \boldsymbol{A}^{\prime} \mathbf{1}_{p} \boldsymbol{\gamma}^{\prime} \boldsymbol{X} \boldsymbol{R}_{2} \boldsymbol{K}_{2}^{\prime}\left(\boldsymbol{K}_{2} \boldsymbol{K}_{2}^{\prime}\right)^{-}+\boldsymbol{T}_{1} \boldsymbol{K}_{2}^{o \prime},
$$

with an arbitrary matrix $\boldsymbol{T}_{1}$. Plugging in the value of $\boldsymbol{B}$ into (7) yields

$$
\left(\boldsymbol{X} \boldsymbol{C}^{\prime o}\left(\boldsymbol{C}^{\prime o}\right)^{\prime}+\boldsymbol{X} \boldsymbol{R}_{2} \boldsymbol{R}_{2}^{\prime}\right) \boldsymbol{Y}^{\prime} \mathbf{1}_{p}-p \boldsymbol{X}\left(\boldsymbol{C}^{\prime o}\left(\boldsymbol{C}^{\prime o}\right)^{\prime}+\boldsymbol{R}_{2} \boldsymbol{Q}_{\boldsymbol{K}_{2}^{\prime}} \boldsymbol{R}_{2}^{\prime}\right) \boldsymbol{X}^{\prime} \boldsymbol{\gamma}-\boldsymbol{X} \boldsymbol{R}_{2} \boldsymbol{P}_{\boldsymbol{K}_{2}^{\prime}} \boldsymbol{R}_{2}^{\prime} \boldsymbol{Y}^{\prime} \mathbf{1}_{p}=\mathbf{0}
$$

Then, we derive the estimators for $\boldsymbol{\gamma}$ and $\boldsymbol{B}$. In the case when $\boldsymbol{X}$ is of full rank, the estimators are obtained by

$$
\begin{aligned}
& \widehat{\boldsymbol{\gamma}}=\frac{1}{p}\left(\boldsymbol{X} \boldsymbol{P} \boldsymbol{X}^{\prime}\right)^{-1} \boldsymbol{X} \boldsymbol{P} \boldsymbol{Y}^{\prime} \mathbf{1}_{p}, \\
& \widehat{\boldsymbol{B}}=\left(\boldsymbol{A}^{\prime} \boldsymbol{A}\right)^{-1} \boldsymbol{A}^{\prime}\left(\boldsymbol{Y}-\frac{1}{p} \mathbf{1}_{p} \mathbf{1}_{p}^{\prime} \boldsymbol{Y} \boldsymbol{P} \boldsymbol{X}^{\prime}\left(\boldsymbol{X} \boldsymbol{P} \boldsymbol{X}^{\prime}\right)^{-1} \boldsymbol{X}\right) \boldsymbol{R}_{2} \boldsymbol{K}_{2}^{\prime}\left(\boldsymbol{K}_{2} \boldsymbol{K}_{2}^{\prime}\right)^{-}+\boldsymbol{T}_{1} \boldsymbol{K}_{2}^{o \prime},
\end{aligned}
$$

with an arbitrary matrix $\boldsymbol{T}_{1}$ and $\boldsymbol{P}=\boldsymbol{C}^{\prime o}\left(\boldsymbol{C}^{\prime o}\right)^{\prime}+\boldsymbol{R}_{2} \boldsymbol{Q}_{\boldsymbol{K}_{2}^{\prime}} \boldsymbol{R}_{2}^{\prime}$. In the general case when $\boldsymbol{X}$ is not necessary of full rank, we get

$$
\begin{aligned}
\widehat{\gamma} & =\frac{1}{p}\left(\boldsymbol{X} \boldsymbol{P} \boldsymbol{X}^{\prime}\right)^{-} \boldsymbol{X} \boldsymbol{P} \boldsymbol{Y}^{\prime} \mathbf{1}_{p}+\left(\boldsymbol{X} \boldsymbol{P} \boldsymbol{X}^{\prime}\right)^{o} \boldsymbol{t}_{2}, \\
\widehat{\boldsymbol{B}} & =\left(\boldsymbol{A}^{\prime} \boldsymbol{A}\right)^{-1} \boldsymbol{A}^{\prime}\left(\boldsymbol{Y}-\frac{1}{p} \mathbf{1}_{p} \mathbf{1}_{p}^{\prime} \boldsymbol{Y} \boldsymbol{P} \boldsymbol{X}^{\prime}\left(\boldsymbol{X} \boldsymbol{P} \boldsymbol{X}^{\prime}\right)^{-} \boldsymbol{X}\right. \\
& \left.-\mathbf{1}_{p} \boldsymbol{t}_{2}^{\prime}\left(\boldsymbol{X} \boldsymbol{P} \boldsymbol{X}^{\prime}\right)^{o^{\prime}} \boldsymbol{X}\right) \boldsymbol{R}_{2} \boldsymbol{K}_{2}^{\prime}\left(\boldsymbol{K}_{2} \boldsymbol{K}_{2}^{\prime}\right)^{-}+\boldsymbol{T}_{1} \boldsymbol{K}_{2}^{o^{\prime}},
\end{aligned}
$$


with an arbitrary vector $\boldsymbol{t}_{2}$ and an arbitrary matrix $\boldsymbol{T}_{1}$. However, there is also information about $\boldsymbol{B}$ and $\boldsymbol{\gamma}$ in $\boldsymbol{V}_{1}$ which we now try to utilize in order to find expressions of $\boldsymbol{T}_{1}$ and $\boldsymbol{t}_{2}$. Recall that

$$
\boldsymbol{V}_{1}=\boldsymbol{Y} \boldsymbol{R}_{1}=\boldsymbol{A} \boldsymbol{B} \boldsymbol{K}_{1}+\mathbf{1}_{p} \boldsymbol{\gamma}^{\prime} \boldsymbol{X} \boldsymbol{R}_{1}+(\boldsymbol{U} \boldsymbol{Z}+\boldsymbol{E}) \boldsymbol{R}_{1}
$$

where $(\boldsymbol{U} \boldsymbol{Z}+\boldsymbol{E}) \boldsymbol{R}_{1} \sim \mathcal{N}_{p, m}\left(\mathbf{0}, \boldsymbol{\Sigma}_{u}+\boldsymbol{\Sigma}_{e}, \boldsymbol{I}_{m}\right)$. Inserting the values of $\boldsymbol{\gamma}$ and $\boldsymbol{B}$ in the model for $\boldsymbol{V}_{1}$ given above, implies

$$
\begin{aligned}
\boldsymbol{V}_{1}= & \boldsymbol{P}_{\boldsymbol{A}} \boldsymbol{Y} \boldsymbol{R}_{2} \boldsymbol{K}_{2}^{\prime}\left(\boldsymbol{K}_{2} \boldsymbol{K}_{2}^{\prime}\right)^{-} \boldsymbol{K}_{1}-\frac{1}{p} \mathbf{1}_{p} \mathbf{1}_{p}^{\prime} \boldsymbol{Y} \boldsymbol{P} \boldsymbol{X}^{\prime}\left(\boldsymbol{X} \boldsymbol{P} \boldsymbol{X}^{\prime}\right)^{-} \boldsymbol{X} \boldsymbol{R}_{2} \boldsymbol{K}_{2}^{\prime}\left(\boldsymbol{K}_{2} \boldsymbol{K}_{2}^{\prime}\right)^{-} \boldsymbol{K}_{1} \\
& -\mathbf{1}_{p} \boldsymbol{t}_{2}^{\prime}\left(\boldsymbol{X} \boldsymbol{P} \boldsymbol{X}^{\prime}\right)^{o^{\prime}} \boldsymbol{X} \boldsymbol{R}_{2} \boldsymbol{K}_{2}^{\prime}\left(\boldsymbol{K}_{2} \boldsymbol{K}_{2}^{\prime}\right)^{-} \boldsymbol{K}_{1}+\boldsymbol{A} \boldsymbol{T}_{1} \boldsymbol{K}_{2}^{o \prime} \boldsymbol{K}_{1} \\
& +\frac{1}{p} \mathbf{1}_{p} \mathbf{1}_{p}^{\prime} \boldsymbol{Y} \boldsymbol{P} \boldsymbol{X}^{\prime}\left(\boldsymbol{X} \boldsymbol{P} \boldsymbol{X}^{\prime}\right)^{-} \boldsymbol{X} \boldsymbol{R}_{1}+\mathbf{1}_{p} \boldsymbol{t}_{2}^{\prime}\left(\boldsymbol{X} \boldsymbol{P} \boldsymbol{X}^{\prime}\right)^{o^{\prime}} \boldsymbol{X} \boldsymbol{R}_{1}+(\boldsymbol{U} \boldsymbol{Z}+\boldsymbol{E}) \boldsymbol{R}_{1}
\end{aligned}
$$

Set

$$
\begin{aligned}
\boldsymbol{V}_{3} & =\boldsymbol{Y} \boldsymbol{R}_{1}-\boldsymbol{P}_{\boldsymbol{A}} \boldsymbol{Y} \boldsymbol{R}_{2} \boldsymbol{K}_{2}^{\prime}\left(\boldsymbol{K}_{2} \boldsymbol{K}_{2}^{\prime}\right)^{-} \boldsymbol{K}_{1} \\
& +\frac{1}{p} \mathbf{1}_{p} \mathbf{1}_{p}^{\prime} \boldsymbol{Y} \boldsymbol{P} \boldsymbol{X}^{\prime}\left(\boldsymbol{X} \boldsymbol{P} \boldsymbol{X}^{\prime}\right)^{-} \boldsymbol{X} \boldsymbol{R}_{2} \boldsymbol{K}_{2}^{\prime}\left(\boldsymbol{K}_{2} \boldsymbol{K}_{2}^{\prime}\right)^{-} \boldsymbol{K}_{1}-\frac{1}{p} \mathbf{1}_{p} \mathbf{1}_{p}^{\prime} \boldsymbol{Y} \boldsymbol{P} \boldsymbol{X}^{\prime}\left(\boldsymbol{X} \boldsymbol{P} \boldsymbol{X}^{\prime}\right)^{-} \boldsymbol{X} \boldsymbol{R}_{1}
\end{aligned}
$$

with model equation ( conditional to given $\boldsymbol{Y} \boldsymbol{R}_{2}$ and $\boldsymbol{Y} \boldsymbol{C}^{\prime o}$ ) equals

$$
\boldsymbol{V}_{3}=\boldsymbol{A} \boldsymbol{T}_{1} \widetilde{\boldsymbol{C}}_{1}+\mathbf{1}_{p} \boldsymbol{t}_{2}^{\prime} \widetilde{\boldsymbol{C}}_{2}+\widetilde{\boldsymbol{E}}
$$

where $\widetilde{\boldsymbol{C}}_{1}=\boldsymbol{K}_{2}^{o \prime} \boldsymbol{K}_{1}, \quad \widetilde{\boldsymbol{C}}_{2}=\left(\boldsymbol{X} \boldsymbol{P} \boldsymbol{X}^{\prime}\right)^{o^{\prime}} \boldsymbol{X} \boldsymbol{R}_{1}-\left(\boldsymbol{X} \boldsymbol{P} \boldsymbol{X}^{\prime}\right)^{\circ \prime} \boldsymbol{X} \boldsymbol{R}_{2} \boldsymbol{K}_{2}^{\prime}\left(\boldsymbol{K}_{2} \boldsymbol{K}_{2}^{\prime}\right)^{-} \boldsymbol{K}_{1}$, and $\widetilde{\boldsymbol{E}}=$ $(\boldsymbol{U} \boldsymbol{Z}+\boldsymbol{E}) \boldsymbol{R}_{1}$. Hence, the model obtained in (11) is an extended multivariate linear growth curve model with a nested subspace condition $\mathcal{C}\left(\mathbf{1}_{p}\right) \subseteq \mathcal{C}(\boldsymbol{A})$ on the within individual design matrices. One can refer to Fujikoshi et al. (1999) and Filipiak and von Rosen (2012) for more details about the extended multivariate linear growth curve model and the corresponding maximum likelihood estimators.

Lemma 4.2. Let $\boldsymbol{V}_{3}$ be defined as in (11). The maximum likelihood estimators for the parameter matrices $\boldsymbol{T}_{1}, \boldsymbol{t}_{2}$ and $\boldsymbol{\Sigma}_{u}$ are given by

$$
\begin{aligned}
& \widehat{\boldsymbol{t}}_{2}=\left(\mathbf{1}_{p}^{\prime} \boldsymbol{S}_{1}^{-1} \mathbf{1}_{p}\right)^{-1}\left(\widetilde{\boldsymbol{C}}_{2} \boldsymbol{Q}_{\widetilde{\boldsymbol{C}}_{1}^{\prime}} \widetilde{\boldsymbol{C}}_{2}^{\prime}\right)^{-} \widetilde{\boldsymbol{C}}_{2} \boldsymbol{Q}_{\widetilde{\boldsymbol{C}}_{1}^{\prime}} \boldsymbol{V}_{3}^{\prime} \boldsymbol{S}_{1}^{-1} \mathbf{1}_{p}+\left(\widetilde{\boldsymbol{C}}_{2} \boldsymbol{Q}_{\widetilde{\boldsymbol{C}}_{1}^{\prime}}\right)^{o^{\prime}} \boldsymbol{t}_{21}^{\prime} \mathbf{1}_{p}, \\
& \widehat{\boldsymbol{T}}_{1}=\left(\boldsymbol{A}^{\prime} \boldsymbol{S}_{2}^{-1} \boldsymbol{A}\right)^{-1} \boldsymbol{A}^{\prime} \boldsymbol{S}_{2}^{-1}\left(\boldsymbol{V}_{3}-\mathbf{1}_{p} \widehat{\boldsymbol{t}}_{2}^{\prime} \widetilde{\boldsymbol{C}}_{2}\right) \widetilde{\boldsymbol{C}}_{1}^{\prime}\left(\widetilde{\boldsymbol{C}}_{1} \widetilde{\boldsymbol{C}}_{1}^{\prime}\right)^{-}+\boldsymbol{A}^{\prime} \boldsymbol{T}_{11} \widetilde{\boldsymbol{C}}_{1}^{o^{\prime}}, \\
& \widehat{\boldsymbol{\Sigma}}_{u}=\frac{1}{m}\left(\boldsymbol{V}_{3}-\boldsymbol{A} \widehat{\boldsymbol{T}}_{1} \widetilde{\boldsymbol{C}}_{1}-\mathbf{1}_{p} \widehat{\boldsymbol{t}}_{2} \widetilde{\boldsymbol{C}}_{2}\right)\left(\boldsymbol{V} 3-\boldsymbol{A} \widehat{\boldsymbol{T}}_{1} \widetilde{\boldsymbol{C}}_{1}-\mathbf{1}_{p} \widehat{\boldsymbol{t}}_{2} \widetilde{\boldsymbol{C}}_{2}\right)^{\prime}-\widehat{\sigma}_{e}^{2} \boldsymbol{I}_{p},
\end{aligned}
$$

for an arbitrary vector $\boldsymbol{t}_{21}$ and an arbitrary matrix $\boldsymbol{T}_{11}$, where $\boldsymbol{S}_{1}=\boldsymbol{V}_{3} \boldsymbol{Q}_{\left(\widetilde{\boldsymbol{C}}_{1}^{\prime}: \widetilde{\boldsymbol{C}}_{2}^{\prime}\right)} \boldsymbol{V}_{3}^{\prime}, \quad \boldsymbol{S}_{2}=$ $\boldsymbol{S}_{1}+\boldsymbol{Q}_{\mathbf{1}_{p}, \boldsymbol{S}_{1}^{-1}} \boldsymbol{V}_{3} \boldsymbol{P}_{\boldsymbol{Q}_{\widetilde{C}_{1}^{\prime}} \widetilde{\boldsymbol{C}}_{2}^{\prime}} \boldsymbol{V}_{3}^{\prime} \boldsymbol{Q}_{\mathbf{1}_{p}, \boldsymbol{S}_{1}^{-1}}^{\prime}$, where it is supposed that $\widehat{\boldsymbol{\Sigma}}_{u}$ is positive definite.

The proof follows similarly to Theorem 1 in Filipiak and von Rosen (2012) in the particular case with two profiles. 
Following Filipiak and von Rosen (2012), from model 111$), \widehat{\boldsymbol{T}}_{1}$ and $\widehat{\boldsymbol{t}}_{2}$ are unique if and only if the matrices $\widetilde{\boldsymbol{C}}_{1}$ and $\widetilde{\boldsymbol{C}}_{2}$ are of full rank, and $\mathcal{C}\left(\widetilde{\boldsymbol{C}}_{1}\right) \cap \mathcal{C}\left(\widetilde{\boldsymbol{C}}_{2}\right)=\{0\}$. However, $\boldsymbol{A} \widehat{\boldsymbol{T}}_{1} \widetilde{\boldsymbol{C}}_{1}$ and $\mathbf{1}_{p} \widehat{\boldsymbol{t}}_{2} \widetilde{\boldsymbol{C}}_{2}$ are unique and hence $\widehat{\boldsymbol{\Sigma}}_{u}$ given in 14 is unique.

With the above calculations, we are now ready to give a theorem which summarizes the main results about estimation of the formulated model.

Theorem 4.1. Consider the model given by (2). Then, estimators of $\boldsymbol{\gamma}, \boldsymbol{B}$ and $\boldsymbol{\Sigma}_{u}$ can be expressed as

$$
\begin{aligned}
\widehat{\boldsymbol{\gamma}}= & \frac{1}{p}\left(\boldsymbol{X} \boldsymbol{P} \boldsymbol{X}^{\prime}\right)^{-} \boldsymbol{X} \boldsymbol{P} \boldsymbol{Y}^{\prime} \mathbf{1}_{p}+\left(\boldsymbol{X} \boldsymbol{P} \boldsymbol{X}^{\prime}\right)^{o} \widehat{\boldsymbol{t}}_{2}, \\
\widehat{\boldsymbol{B}}= & \left(\boldsymbol{A}^{\prime} \boldsymbol{A}\right)^{-1} \boldsymbol{A}^{\prime}\left(\boldsymbol{Y}-\frac{1}{p} \mathbf{1}_{p} \mathbf{1}_{p}^{\prime} \boldsymbol{Y} \boldsymbol{P} \boldsymbol{X}^{\prime}\left(\boldsymbol{X} \boldsymbol{P} \boldsymbol{X}^{\prime}\right)^{-} \boldsymbol{X}-\mathbf{1}_{p} \widehat{\boldsymbol{t}}_{2}^{\prime}\left(\boldsymbol{X} \boldsymbol{P} \boldsymbol{X}^{\prime}\right)^{o^{\prime}} \boldsymbol{X}\right) \boldsymbol{R}_{2} \boldsymbol{K}_{2}^{\prime}\left(\boldsymbol{K}_{2} \boldsymbol{K}_{2}^{\prime}\right)^{-} \\
& +\widehat{\boldsymbol{T}}_{1} \boldsymbol{K}_{2}^{o \prime}, \\
\widehat{\boldsymbol{\Sigma}}_{u}= & \frac{1}{m}\left(\boldsymbol{V}_{3}-\boldsymbol{A} \widehat{\boldsymbol{T}}_{1} \widetilde{\boldsymbol{C}}_{1}-\mathbf{1}_{p} \widehat{\boldsymbol{t}}_{2}^{\prime} \widetilde{\boldsymbol{C}}_{2}\right)\left(\boldsymbol{V}_{3}-\boldsymbol{A} \widehat{\boldsymbol{T}}_{1} \widetilde{\boldsymbol{C}}_{1}-\mathbf{1}_{p} \widehat{\boldsymbol{t}}_{2}^{\prime} \widetilde{\boldsymbol{C}}_{2}\right)^{\prime}-\boldsymbol{\Sigma}_{e},
\end{aligned}
$$

where $\widehat{\boldsymbol{t}}_{2}$ and $\widehat{\boldsymbol{T}}_{1}$ are given by 1 (2) and $(13)$, respectively and it is assumed that $\widehat{\boldsymbol{\Sigma}}_{u}$ is positive definite.

From Theorem 4.1 the estimators $\widehat{\boldsymbol{\gamma}}$ and $\widehat{\boldsymbol{B}}$ depend on an arbitrary vector $\widehat{\boldsymbol{t}}_{2}$ and an arbitrary matrix $\widehat{\boldsymbol{T}}_{1}$, respectively. However, the linear combinations $\boldsymbol{A B H C}$ and $\mathbf{1}_{p} \boldsymbol{\gamma}^{\prime} \boldsymbol{X}$ are uniquely estimated by $\boldsymbol{A} \widehat{\boldsymbol{B}} \boldsymbol{H} \boldsymbol{C}$ and $\mathbf{1}_{p} \widehat{\boldsymbol{\gamma}}^{\prime} \boldsymbol{X}$, respectively. Thus, the estimator of the mean from the model defined in 22 is given by $\boldsymbol{A} \widehat{\boldsymbol{B}} \boldsymbol{H} \boldsymbol{C}+\mathbf{1}_{p} \widehat{\boldsymbol{\gamma}}^{\prime} \boldsymbol{X}$. We note that all the estimators $\widehat{\boldsymbol{\gamma}}, \widehat{\boldsymbol{B}}, \widehat{\boldsymbol{\Sigma}}_{u}$ are explicit estimators. The next corollaries follow from Theorem 4.1 .

Corollary 4.1. Consider the model defined by (2) and suppose that $p=q$. Then, the within individuals design matrix $\boldsymbol{A}$ is non singular and the estimators for $\boldsymbol{\gamma}, \boldsymbol{B}$ and $\boldsymbol{\Sigma}_{u}$ are given by

$$
\begin{aligned}
\widehat{\boldsymbol{\gamma}}= & \frac{1}{p}\left(\boldsymbol{X} \boldsymbol{P} \boldsymbol{X}^{\prime}\right)^{-} \boldsymbol{X} \boldsymbol{P} \boldsymbol{Y}^{\prime} \mathbf{1}_{p}+\left(\boldsymbol{X} \boldsymbol{P} \boldsymbol{X}^{\prime}\right)^{o} \widehat{\boldsymbol{t}}_{3}, \\
\widehat{\boldsymbol{B}}= & \boldsymbol{A}^{-1}\left(\boldsymbol{Y} \boldsymbol{R}_{2} \boldsymbol{K}_{2}^{\prime}\left(\boldsymbol{K}_{2} \boldsymbol{K}_{2}^{\prime}\right)^{-}-\frac{1}{p} \mathbf{1}_{p} \mathbf{1}_{p}^{\prime} \boldsymbol{Y} \boldsymbol{P} \boldsymbol{X}^{\prime}\left(\boldsymbol{X} \boldsymbol{P} \boldsymbol{X}^{\prime}\right)^{-} \boldsymbol{X} \boldsymbol{R}_{2} \boldsymbol{K}_{2}^{\prime}\left(\boldsymbol{K}_{2} \boldsymbol{K}_{2}^{\prime}\right)^{-}\right. \\
& \left.-\mathbf{1}_{p} \widehat{\boldsymbol{t}}_{3}\left(\boldsymbol{X} \boldsymbol{P} \boldsymbol{X}^{\prime}\right)^{o} \boldsymbol{X} \boldsymbol{R}_{2} \boldsymbol{K}_{2}^{\prime}\left(\boldsymbol{K}_{2} \boldsymbol{K}_{2}^{\prime}\right)^{-}\right)+\widehat{\boldsymbol{T}}_{4} \boldsymbol{K}_{2}^{o \prime}, \\
\widehat{\boldsymbol{\Sigma}}_{u}= & \frac{1}{m}\left(\boldsymbol{V}_{4}-\widehat{\boldsymbol{T}}_{4} \widetilde{\boldsymbol{C}}_{1}-\mathbf{1}_{p} \widehat{\boldsymbol{t}}_{3}^{\prime} \widetilde{\boldsymbol{C}}_{2}\right)\left(\boldsymbol{V}_{4}-\widehat{\boldsymbol{T}}_{4} \widetilde{\boldsymbol{C}}_{1}-\mathbf{1}_{p} \widehat{\boldsymbol{t}}_{3}^{\prime} \widetilde{\boldsymbol{C}}_{2}\right)^{\prime}-\boldsymbol{\Sigma}_{e},
\end{aligned}
$$

where

$$
\begin{aligned}
\boldsymbol{V}_{4}= & \boldsymbol{Y} \boldsymbol{R}_{1}-\frac{1}{p} \mathbf{1}_{p} \mathbf{1}_{p}^{\prime} \boldsymbol{Y} \boldsymbol{P} \boldsymbol{X}^{\prime}\left(\boldsymbol{X} \boldsymbol{P} \boldsymbol{X}^{\prime}\right)^{-} \boldsymbol{X} \boldsymbol{R}_{1} \\
& +\frac{1}{p} \mathbf{1}_{p} \mathbf{1}_{p}^{\prime} \boldsymbol{Y} \boldsymbol{P} \boldsymbol{X}^{\prime}\left(\boldsymbol{X} \boldsymbol{P} \boldsymbol{X}^{\prime}\right)^{-} \boldsymbol{X} \boldsymbol{R}_{2} \boldsymbol{K}_{2}^{\prime}\left(\boldsymbol{K}_{2} \boldsymbol{K}_{2}^{\prime}\right)^{-} \boldsymbol{K}_{1}-\boldsymbol{Y} \boldsymbol{R}_{2} \boldsymbol{K}_{2}^{\prime}\left(\boldsymbol{K}_{2} \boldsymbol{K}_{2}^{\prime}\right)^{-} \boldsymbol{K}_{1} \\
\widehat{\boldsymbol{t}}_{3}= & \left(\mathbf{1}_{p}^{\prime} \boldsymbol{S}_{3}^{-1} \mathbf{1}_{p}\right)^{-1}\left(\widetilde{\boldsymbol{C}}_{2} \boldsymbol{Q}_{\widetilde{\boldsymbol{C}}_{1}^{\prime}} \widetilde{\boldsymbol{C}}_{2}^{\prime}\right)^{-} \widetilde{\boldsymbol{C}}_{2} \boldsymbol{Q}_{\widetilde{\boldsymbol{C}}_{1}^{\prime}} \boldsymbol{V}_{4}^{\prime} \boldsymbol{S}_{3}^{-1} \mathbf{1}_{p}+\left(\widetilde{\boldsymbol{C}}_{2} \boldsymbol{Q}_{\widetilde{\boldsymbol{C}}_{1}^{\prime}}\right)^{o} \boldsymbol{t}_{31}^{\prime} \mathbf{1}_{p} \\
\widehat{\boldsymbol{T}}_{4}= & \boldsymbol{A}^{-1}\left(\boldsymbol{V}_{4}-\mathbf{1}_{p} \widehat{\boldsymbol{t}}_{3}^{\prime} \widetilde{\boldsymbol{C}}_{2}\right) \widetilde{\boldsymbol{C}}_{1}^{\prime}\left(\widetilde{\boldsymbol{C}}_{1} \widetilde{\boldsymbol{C}}_{1}^{\prime}\right)^{-}+\boldsymbol{A}^{\prime} \boldsymbol{T}_{41} \widetilde{\boldsymbol{C}}_{1}^{o} \\
\boldsymbol{S}_{3}= & \boldsymbol{V}_{4} \boldsymbol{Q}_{\left(\widetilde{\boldsymbol{C}}_{1}^{\prime}: \widetilde{\boldsymbol{C}}_{2}^{\prime}\right)} \boldsymbol{V}_{4}^{\prime}
\end{aligned}
$$


for an arbitrary vector $\boldsymbol{t}_{31}$ and an arbitrary matrix $\boldsymbol{T}_{41}$ with $\widehat{\boldsymbol{\Sigma}}_{u}$ assumed to be positive definite.

Corollary 4.2. Consider the model defined by (2) and suppose that the matrix $\boldsymbol{X}$ of covariates is of full rank. Then, the estimators for $\boldsymbol{\gamma}, \boldsymbol{B}$ and $\boldsymbol{\Sigma}_{u}$ are given by

$$
\begin{aligned}
\widehat{\gamma} & =\frac{1}{p}\left(\boldsymbol{X} \boldsymbol{P} \boldsymbol{X}^{\prime}\right)^{-1} \boldsymbol{X} \boldsymbol{P} \boldsymbol{Y}^{\prime} \mathbf{1}_{p}, \\
\widehat{\boldsymbol{B}} & =\left(\boldsymbol{A}^{\prime} \boldsymbol{A}\right)^{-1} \boldsymbol{A}^{\prime}\left(\boldsymbol{Y}-\frac{1}{p} \mathbf{1}_{p} \mathbf{1}_{p}^{\prime} \boldsymbol{Y} \boldsymbol{P} \boldsymbol{X}^{\prime}\left(\boldsymbol{X} \boldsymbol{P} \boldsymbol{X}^{\prime}\right)^{-1} \boldsymbol{X}\right) \boldsymbol{R}_{2} \boldsymbol{K}_{2}^{\prime}\left(\boldsymbol{K}_{2} \boldsymbol{K}_{2}^{\prime}\right)^{-}+\widehat{\boldsymbol{T}} \boldsymbol{K}_{2}^{o \prime}, \\
\widehat{\boldsymbol{\Sigma}}_{u} & =\frac{1}{m}\left(\boldsymbol{V}_{3}-\boldsymbol{A} \widehat{\boldsymbol{T}} \widetilde{\boldsymbol{C}}_{1}\right)\left(\boldsymbol{V}_{3}-\boldsymbol{A} \widehat{\boldsymbol{T}} \widetilde{\boldsymbol{C}}_{1}\right)^{\prime}-\boldsymbol{\Sigma}_{e},
\end{aligned}
$$

where

$$
\begin{aligned}
\widehat{\boldsymbol{T}} & =\left(\boldsymbol{A}^{\prime} \boldsymbol{S}^{-1} \boldsymbol{A}\right)^{-1} \boldsymbol{A}^{\prime} \boldsymbol{S}^{-1} \boldsymbol{V}_{3} \widetilde{\boldsymbol{C}}_{1}^{\prime}\left(\widetilde{\boldsymbol{C}}_{1} \widetilde{\boldsymbol{C}}_{1}^{\prime}\right)^{-}+\boldsymbol{A}^{\prime} \boldsymbol{T}_{12} \widetilde{\boldsymbol{C}}_{1}^{o}, \\
\boldsymbol{S} & =\boldsymbol{V}_{3} \boldsymbol{Q}_{\widetilde{\boldsymbol{C}}_{1}^{\prime}} \boldsymbol{V}_{3}^{\prime},
\end{aligned}
$$

for an arbitrary matrix $\boldsymbol{T}_{12}$.

\section{Prediction of random effects and target small area means}

Next we turn to the second focus of this article, namely to perform prediction. The small area means $\boldsymbol{\mu}_{i}$ in a given $i$-th area, are defined as the conditional mean given the random area effects $\boldsymbol{u}_{i}$ (Battese et al., 1988). That is $\boldsymbol{\mu}_{i}=\mathbb{E}\left[\boldsymbol{Y}_{i} \mid \boldsymbol{u}_{i}\right]$. Thus, prediction of random effects are needed in the prediction of small area means. Robinson (1991) discusses the need of prediction of random effects and summarizes the theory of the best linear unbiased predictor (BLUP) with examples and applications. The idea is to predict the values of unobservable random variables based on some realized, observed values. Moreover, Searle et al. (2009) have extensively studied the prediction of random variables under general mixed linear models. As stated by these authors, while the best linear unbiased estimator for fixed parameter is the estimator with the minimum variance, the best linear unbiased predictor for a realized value of a random variable is the estimator with minimum mean-squared error among the class of linear estimators. In this section, we adopt a third approach developed by Henderson (1973) for prediction of random effects which consists of maximizing the joint density of the observable and non-observable random variable.

Consider the model in (2) given by

$$
\boldsymbol{Y}=\boldsymbol{A B H C}+\mathbf{1}_{p} \boldsymbol{\gamma}^{\prime} \boldsymbol{X}+\boldsymbol{U} \boldsymbol{Z}+\boldsymbol{E},
$$

and according to Henderson (1973) we shall maximize the joint density $f(\boldsymbol{Y}, \boldsymbol{U})$ with respect 
to $\boldsymbol{U}$ assuming the other parameters $\boldsymbol{B}, \boldsymbol{\gamma}$ and $\boldsymbol{\Sigma}_{u}$ to be known. We get

$$
\begin{aligned}
f(\boldsymbol{Y}, \boldsymbol{U})= & f(\boldsymbol{U}) f(\boldsymbol{Y} \mid \boldsymbol{U})=\lambda \exp \left\{-\frac{1}{2} \operatorname{tr}\left\{\boldsymbol{U}^{\prime} \boldsymbol{\Sigma}_{u}^{-1} \boldsymbol{U}\right\}\right\} \\
& \times \exp \left\{-\frac{1}{2} \operatorname{tr}\left\{\boldsymbol{\Sigma}_{e}^{-1}\left(\boldsymbol{Y}-\boldsymbol{A} \boldsymbol{B} \boldsymbol{H} \boldsymbol{C}-\mathbf{1}_{p} \boldsymbol{\gamma}^{\prime} \boldsymbol{X}-\boldsymbol{U} \boldsymbol{Z}\right)\right.\right. \\
& \left.\left.\times\left(\boldsymbol{Y}-\boldsymbol{A} \boldsymbol{B} \boldsymbol{H} \boldsymbol{C}-\mathbf{1}_{p} \boldsymbol{\gamma}^{\prime} \boldsymbol{X}-\boldsymbol{U} \boldsymbol{Z}\right)^{\prime}\right\}\right\}
\end{aligned}
$$

where $\lambda$ is a known constant. Then, the estimating equation for $\boldsymbol{U}$ equals

$$
\boldsymbol{\Sigma}_{e}^{-1}\left(\boldsymbol{Y}-\boldsymbol{A B} \boldsymbol{H} \boldsymbol{C}-\mathbf{1}_{p} \gamma^{\prime} \boldsymbol{X}-\boldsymbol{U} \boldsymbol{Z}\right) \boldsymbol{Z}^{\prime}-\boldsymbol{\Sigma}_{u}^{-1} \boldsymbol{U}=\mathbf{0}
$$

which gives

$$
\widetilde{\boldsymbol{U}}=\left(\boldsymbol{\Sigma}_{e} \boldsymbol{\Sigma}_{u}^{-1}+\boldsymbol{I}_{p}\right)^{-1}\left(\boldsymbol{Y}-\boldsymbol{A B} \boldsymbol{H} \boldsymbol{C}-\mathbf{1}_{p} \boldsymbol{\gamma}^{\prime} \boldsymbol{X}\right) \boldsymbol{Z}^{\prime}
$$

Since the parameters $\boldsymbol{B}, \boldsymbol{\gamma}$, and $\boldsymbol{\Sigma}_{u}$ are unknown, they are replaced by their estimators obtained in the previous section in Theorem 4.1. Although these estimators could be derived from maximizing the joint density $f(\boldsymbol{Y}, \boldsymbol{U})$ with respect to all parameters, the obtained estimating equations lead only to implicit estimators, i.e., only numerical expressions can be obtained. Therefore, according to our approach we get the following theorem about the prediction of random effects.

Theorem 5.1. Consider the model defined by (2). The predicted random effects are given by

$$
\widehat{\boldsymbol{U}}=\left(\boldsymbol{\Sigma}_{e} \widehat{\boldsymbol{\Sigma}}_{u}^{-1}+\boldsymbol{I}_{p}\right)^{-1}\left(\boldsymbol{Y}-\boldsymbol{A} \widehat{\boldsymbol{B}} \boldsymbol{H} \boldsymbol{C}-\mathbf{1}_{p} \widehat{\boldsymbol{\gamma}}^{\prime} \boldsymbol{X}\right) \boldsymbol{Z}^{\prime}
$$

where $\widehat{\boldsymbol{\gamma}}, \widehat{\boldsymbol{B}}$ and $\widehat{\boldsymbol{\Sigma}}_{u}$ are presented in Theorem 4.1 .

Estimating the quantities of interest namely the small area means is equivalent to predicting small area means of non sampled values, given the sample data and auxiliary data (Rao, 2003; Royall, 1988). Following Prasad and Rao (1990), Ghosh and Rao (1994), Battese et al. (1988), the target vector in small area $i$, which elements are area means at each time point, is given by

$$
\begin{aligned}
\widehat{\boldsymbol{\mu}}_{i} & =\frac{f_{i}}{n_{i}} \boldsymbol{Y}_{i}^{(s)} \mathbf{1}_{n_{i}}+\frac{1-f_{i}}{N_{i}-n_{i}} \widehat{\boldsymbol{Y}}_{i}^{(r)} \mathbf{1}_{N_{i}-n_{i}} \\
& =\frac{1}{N_{i}}\left(\boldsymbol{Y}_{i}^{(s)} \mathbf{1}_{n_{i}}+\left(\boldsymbol{A} \widehat{\boldsymbol{B}} \boldsymbol{C}_{i}^{(r)}+\mathbf{1}_{p} \widehat{\boldsymbol{\gamma}}^{\prime} \boldsymbol{X}_{i}^{(r)}+\widehat{\boldsymbol{u}}_{i} \boldsymbol{z}_{i}^{(r)^{\prime}}\right) \mathbf{1}_{N_{i}-n_{i}}\right),
\end{aligned}
$$

where $\boldsymbol{Y}_{i}^{(s)}: p \times n_{i}$ stands for the matrix of observed sample data, $\widehat{\boldsymbol{Y}}_{i}^{(r)}: p \times N_{i}-n_{i}$ corresponds to the predicted matrix of non sampled data, with $\boldsymbol{X}_{i}^{(r)}, \boldsymbol{C}_{i}^{(r)}$ and $\boldsymbol{z}_{i}^{(r)}$ being the corresponding matrix of auxiliary information, design matrix and design vector for non sampled units, respectively. Moreover, the predicted vector $\widehat{\boldsymbol{u}}_{i}$ is the $i$-th column of the predicted matrix $\widehat{\boldsymbol{U}}$ and $1-f_{i}$ 
is the finite population correction factor with $f_{i}=\frac{n_{i}}{N_{i}}$ the fraction of the population that is sampled.

The corresponding small area means at each time point for each one of $k$ group units is then given by

$$
\widehat{\boldsymbol{\mu}}_{i g}^{(t)}=\frac{1}{N_{i g}}\left(\boldsymbol{Y}_{i g}^{(s)} \mathbf{1}_{n_{i g}}+\left(\boldsymbol{A} \widehat{\boldsymbol{\beta}}_{g} \mathbf{1}_{N_{i g}-n_{i g}}^{\prime}+\mathbf{1}_{p} \widehat{\boldsymbol{\gamma}}^{\prime} \boldsymbol{X}_{i g}^{(r)}+\widehat{\boldsymbol{u}}_{i} \boldsymbol{z}_{i g}^{(r)^{\prime}}\right) \mathbf{1}_{N_{i g}-n_{i g}}\right), \quad g=1, \ldots, k,
$$

where $\widehat{\boldsymbol{\gamma}}, \widehat{\boldsymbol{u}}_{i}$ and $\widehat{\boldsymbol{\beta}}_{g}$ are estimators computed from Theorem 4.1 and Theorem 5.1 respectively using observed data and $\widehat{\boldsymbol{\beta}}_{g}$ is the column of the estimated parameter matrix $\widehat{\boldsymbol{B}}$ for the corresponding group $g$.

The target small area means for each group $g$ across all time points can be derived by

$$
\widehat{\mu}_{i g}=\frac{1}{p} \mathbf{1}_{p}^{\prime} \widehat{\boldsymbol{\mu}}_{i g}^{(t)} .
$$

Now, we are ready to give the next theorem which includes some of the main results.

Theorem 5.2. Given the multivariate linear regression model defined in (2), the target small area means at each time point are elements of the vectors

$$
\widehat{\boldsymbol{\mu}}_{i}=\frac{1}{N_{i}}\left(\boldsymbol{Y}_{i}^{(s)} \mathbf{1}_{n_{i}}+\left(\boldsymbol{A} \widehat{\boldsymbol{B}} \boldsymbol{C}_{i}^{(r)}+\mathbf{1}_{p} \widehat{\boldsymbol{\gamma}}^{\prime} \boldsymbol{X}_{i}^{(r)}+\widehat{\boldsymbol{u}}_{i} \boldsymbol{z}_{i}^{(r)^{\prime}}\right) \mathbf{1}_{N_{i}-n_{i}}\right), i=1, \cdots, m,
$$

and the small area means at each time point for each group units are given by

$$
\begin{aligned}
\widehat{\boldsymbol{\mu}}_{i g}^{(t)}= & \frac{1}{N_{i g}}\left(\boldsymbol{Y}_{i g}^{(s)} \mathbf{1}_{n_{i g}}+\left(\boldsymbol{A} \widehat{\boldsymbol{\beta}}_{g} \mathbf{1}_{N_{i g}-n_{i g}}^{\prime}+\mathbf{1}_{p} \widehat{\boldsymbol{\gamma}}^{\prime} \boldsymbol{X}_{i g}^{(r)}+\widehat{\boldsymbol{u}}_{i} \boldsymbol{z}_{i g}^{(r)^{\prime}}\right) \mathbf{1}_{N_{i g}-n_{i g}}\right), \\
& g=1, \ldots, k, t=1, \ldots, p .
\end{aligned}
$$

The target small area means for each group across all time points are given by

$$
\widehat{\mu}_{i g}=\frac{1}{p} \mathbf{1}_{p}^{\prime} \widehat{\boldsymbol{\mu}}_{i g}^{(t)} .
$$

\section{Parametric Bootstrap for Mean-Squared Errors (MSE)}

The derivation of an analytic expression for the MSE of the predicted small area means presented in Theorem 5.2 is not an easy task. Instead, the estimation of MSE can be performed using the parametric bootstrap method which has been proved to be successful in many situations. Following Hall and Maiti (2006); González-Manteiga et al. (2008); Jiongo et al. (2013), the following steps describe the parametric bootstrap method which can be used for estimating the MSE of the predicted small area means $\widehat{\boldsymbol{\mu}}_{i g}^{(t)}$.

1) From the sample data, calculate estimators $\widehat{\boldsymbol{B}}, \widehat{\boldsymbol{\gamma}}$ and $\widehat{\boldsymbol{\Sigma}}_{u}$ for $\boldsymbol{B}, \boldsymbol{\gamma}$ and $\boldsymbol{\Sigma}_{u}$, respectively, as well as $\widehat{U}$; 
2) Generate independent area-specific random effects $\boldsymbol{u}_{i}^{*}, i=1, \ldots, m$ from $\boldsymbol{u}_{i}^{*} \sim \mathcal{N}_{p}\left(\mathbf{0}, \widehat{\boldsymbol{\Sigma}}_{u}\right)$;

3) Generate bootstrap population $\left\{\boldsymbol{Y}_{i}^{*}\right\}: p \times N_{i}, i=1, \ldots, m$ from the model

$$
\boldsymbol{Y}_{i}^{*}=\boldsymbol{A} \widehat{\boldsymbol{B}} \boldsymbol{C}_{i}+\mathbf{1}_{p} \widehat{\boldsymbol{\gamma}}^{\prime} \boldsymbol{X}_{i}+\boldsymbol{u}_{i}^{*} \boldsymbol{z}_{i}^{\prime}+\boldsymbol{E}_{i}^{*}, \quad i=1, \ldots, m,
$$

where $\boldsymbol{E}_{i}^{*}=\left(\boldsymbol{e}_{i 1}^{*}, \ldots, \boldsymbol{e}_{i m}^{*}\right)^{\prime}$ with $\boldsymbol{e}_{i j}^{*} \sim \mathcal{N}_{p}\left(0, \sigma_{e}^{2} \boldsymbol{I}_{p}\right), j=1, \ldots, N_{i}$;

4) Compute the target small area means $\boldsymbol{\mu}_{i g}^{(t) *}$ for the bootstrap population from step 3;

5) Fit the model to bootstrap sample $\left\{\boldsymbol{Y}_{i}^{*}\right\}: p \times n_{i}$ and calculate $\widehat{\boldsymbol{B}}^{*}, \widehat{\boldsymbol{\gamma}}^{*}, \widehat{\boldsymbol{\Sigma}}_{u}^{*}$ and derive the corresponding bootstrap area means $\widehat{\boldsymbol{\mu}}_{i g}^{(t) *}$ using these new estimators;

6) Repeat steps 2) - 5) $R$ times and compute the estimated MSE by

$$
\widehat{M S E}\left(\widehat{\boldsymbol{\mu}}_{i g}^{(t)}\right)=\frac{1}{R} \sum_{r=1}^{R}\left(\widehat{\boldsymbol{\mu}}_{i g}^{(t) *}(r)-\boldsymbol{\mu}_{i g}^{(t) *}(r)\right)\left(\widehat{\boldsymbol{\mu}}_{i g}^{(t) *}(r)-\boldsymbol{\mu}_{i g}^{(t) *}(r)\right)^{\prime} .
$$

\section{$7 \quad$ Simulation study}

In this section we describe the simulation experiments carried out for analyzing the accuracy of predicted small area means and the estimated MSE. We consider a $p=4$ times repeated survey on a variable of interest from a population having $m=5,10,30$ small areas and $k=3$ group units with three covariables. We generate a sample of size $n=240$ from a finite population of size $N=600$. Recall that $n_{i g}, i=1, \ldots, m, g=1, \ldots, k$ denotes the sample size from the $i$-th small area in the $g$-th group unit. Let $n_{i g}^{\prime}$ denotes the corresponding sizes of non sampled units. For simplicity, we consider a balanced size for all small areas with the same number of group units. The following Table 1 and Table 2 give the sizes of sampled and non sampled units from each area and per group units.

Table 1: Sizes of sampled units in small areas used in simulation

\begin{tabular}{c|ccccc}
\hline Area & Group 1 & Group 2 & Group 3 & Total sampled & Total population \\
$m$ & $n_{i 1}$ & $n_{i 2}$ & $n_{i 3}$ & $n_{i}$ & $N_{i}$ \\
\hline 5 & 15 & 16 & 17 & 48 & 120 \\
10 & 7 & 8 & 9 & 24 & 60 \\
30 & 2 & 3 & 3 & 8 & 20
\end{tabular}


Table 2: Sizes of non sampled units in small areas used in simulation

\begin{tabular}{c|cccc}
\hline $\begin{array}{c}\text { Area } \\
m\end{array}$ & $\begin{array}{c}\text { Group 1 } \\
n_{i 1}^{\prime}\end{array}$ & $\begin{array}{c}\text { Group 2 } \\
n_{i 2}^{\prime}\end{array}$ & $\begin{array}{c}\text { Group 3 } \\
n_{i 3}^{\prime}\end{array}$ & $\begin{array}{c}\text { Total non sampled } \\
N_{i}-n_{i}\end{array}$ \\
\hline 5 & 23 & 24 & 25 & 72 \\
10 & 11 & 12 & 13 & 36 \\
30 & 3 & 4 & 5 & 12
\end{tabular}

The design matrices are chosen to be

$$
\begin{array}{r}
\boldsymbol{A}=\left(\begin{array}{ll}
1 & 1 \\
1 & 2 \\
1 & 3 \\
1 & 4
\end{array}\right), \boldsymbol{C}=\left(\begin{array}{ccc}
\boldsymbol{C}_{1} & & \mathbf{0} \\
& \ddots & \\
\mathbf{0} & & \boldsymbol{C}_{m}
\end{array}\right), \text { for } \boldsymbol{C}_{i}=\left(\mathbf{1}_{n_{i 1}}^{\prime} \otimes\left(\begin{array}{l}
1 \\
0 \\
0
\end{array}\right): \mathbf{1}_{n_{i 2}}^{\prime} \otimes\left(\begin{array}{l}
0 \\
1 \\
0
\end{array}\right): \mathbf{1}_{n_{i 3}}^{\prime} \otimes\left(\begin{array}{l}
0 \\
0 \\
1
\end{array}\right)\right), \\
i=1, \cdots, m,
\end{array}
$$

where the symbol $\otimes$ denotes the Kronecker product. The parameter matrices, the sampling variance and the covariance for the random effects are

$$
\boldsymbol{B}=\left(\begin{array}{ccc}
8 & 9 & 10 \\
11 & 12 & 13
\end{array}\right), \quad \gamma=\left(\begin{array}{l}
1 \\
2 \\
3
\end{array}\right), \quad \sigma_{e}^{2}=0.16 \quad \text { and } \quad \boldsymbol{\Sigma}_{u}=\left(\begin{array}{llll}
4.1 & 1.8 & 1.2 & 2.4 \\
1.8 & 3.6 & 2.4 & 1.4 \\
1.2 & 2.4 & 6.0 & 2.2 \\
2.4 & 1.4 & 2.2 & 9.6
\end{array}\right)
$$

Then, the sample data are randomly generated following

$$
\operatorname{vec}(\boldsymbol{Y}) \sim \mathcal{N}_{p n}\left(\operatorname{vec}\left(\boldsymbol{A B H}+\mathbf{1}_{p} \boldsymbol{\gamma}^{\prime} \boldsymbol{X}\right), \boldsymbol{\Sigma}\right)
$$

where

$$
\begin{aligned}
& \boldsymbol{\Sigma}=\boldsymbol{Z}^{\prime} \boldsymbol{Z} \otimes \boldsymbol{\Sigma}_{u}+\boldsymbol{I}_{N} \otimes \boldsymbol{\Sigma}_{e}, \quad \boldsymbol{H}=\underbrace{\left[\boldsymbol{I}_{3}: \cdots: \boldsymbol{I}_{3}\right]}_{m \text { times }}, \\
& \boldsymbol{Z}=\left(\begin{array}{ccc}
\boldsymbol{z}_{1}^{\prime} & & \mathbf{0} \\
& \ddots & \\
\mathbf{0} & & \boldsymbol{z}_{m}^{\prime}
\end{array}\right)=\left(\begin{array}{ccc}
\frac{1}{\sqrt{n_{1}}} \mathbf{1}_{n_{1}}^{\prime} & & \mathbf{0} \\
& \ddots & \\
\mathbf{0} & & \frac{1}{\sqrt{n_{m}}} \mathbf{1}_{n_{m}}^{\prime}
\end{array}\right),
\end{aligned}
$$

and $\operatorname{vec}($.$) is the column-wise vectorization operator. The matrix of covariates \boldsymbol{X}$ in 15 is randomly generated as uniformly distributed on the interval $[0,1]$ and then taken as fixed.

Using MATLAB Version 9.0.0.341360 (The MathWorks, Inc. USA), the simulations were repeated $R=500$ times using the formulas presented in Theorem 5.2 and the parametric bootstrap procedure presented in Section 6. Hereafter are the obtained results. 
The averages of the obtained estimates for the parameters of interest equal

$$
\widehat{\boldsymbol{\gamma}}=\left(\begin{array}{l}
1.04 \\
1.91 \\
2.97
\end{array}\right), \quad \widehat{\boldsymbol{B}}=\left(\begin{array}{ccc}
8.03 & 9.03 & 10.03 \\
11.02 & 12.00 & 13.00
\end{array}\right), \quad \widehat{\boldsymbol{\Sigma}}_{u}=\left(\begin{array}{cccc}
4.13 & 1.70 & 1.09 & 2.58 \\
1.70 & 3.58 & 2.45 & 1.54 \\
1.09 & 2.45 & 6.10 & 2.19 \\
2.58 & 1.54 & 2.19 & 9.50
\end{array}\right) \text {. }
$$

The estimates are in complete agreement with original parameter values showing that the estimation approach works very well. The predicted small area means and corresponding MSE obtained by parametric bootstrap method are presented bellow

- For $m=5$ and $i=1$

$$
\begin{aligned}
& \widehat{\boldsymbol{\mu}}_{11}^{(t)}=\left(\begin{array}{c}
4.288 \\
6.553 \\
8.859 \\
11.003
\end{array}\right), \quad \widehat{\boldsymbol{\mu}}_{12}^{(t)}=\left(\begin{array}{c}
4.728 \\
7.199 \\
9.711 \\
12.041
\end{array}\right), \quad \widehat{\boldsymbol{\mu}}_{13}^{(t)}=\left(\begin{array}{c}
5.128 \\
7.831 \\
10.533 \\
13.050
\end{array}\right), \begin{array}{l}
\widehat{\mu}_{11}=7.676 \\
\widehat{\mu}_{12}=8.420 \\
\widehat{\mu}_{13}=9.136
\end{array} \\
& \operatorname{MSE}\left(\widehat{\boldsymbol{\mu}}_{11}^{(t)}\right)=\left(\begin{array}{llll}
1.228 & 1.234 & 1.252 & 1.237 \\
1.234 & 1.256 & 1.281 & 1.266 \\
1.252 & 1.281 & 1.326 & 1.309 \\
1.237 & 1.266 & 1.309 & 1.320
\end{array}\right), \operatorname{MSE}\left(\widehat{\boldsymbol{\mu}}_{12}^{(t)}\right)=\left(\begin{array}{llll}
1.186 & 1.192 & 1.213 & 1.197 \\
1.192 & 1.215 & 1.242 & 1.227 \\
1.213 & 1.242 & 1.288 & 1.270 \\
1.197 & 1.227 & 1.270 & 1.280
\end{array}\right) \\
& \operatorname{MSE}\left(\widehat{\boldsymbol{\mu}}_{13}^{(t)}\right)=\left(\begin{array}{cccc}
1.155 & 1.159 & 1.177 & 1.160 \\
1.159 & 1.178 & 1.204 & 1.186 \\
1.177 & 1.204 & 1.247 & 1.228 \\
1.160 & 1.186 & 1.228 & 1.2350
\end{array}\right), \quad \begin{array}{l}
M S E\left(\widehat{\mu}_{11}\right)=1.268 \\
M S E\left(\widehat{\mu}_{12}\right)=1.195 \\
M S E\left(\widehat{\mu}_{13}\right)=1.187
\end{array}
\end{aligned}
$$

- For $m=10$ and $i=1$

$$
\begin{aligned}
& \widehat{\boldsymbol{\mu}}_{11}^{(t)}=\left(\begin{array}{l}
2.255 \\
3.394 \\
4.379 \\
5.448
\end{array}\right), \quad \widehat{\boldsymbol{\mu}}_{12}^{(t)}=\left(\begin{array}{l}
2.400 \\
3.649 \\
4.737 \\
5.909
\end{array}\right), \quad \widehat{\boldsymbol{\mu}}_{13}^{(t)}=\left(\begin{array}{l}
2.571 \\
3.910 \\
5.123 \\
6.386
\end{array}\right), \quad \begin{array}{l}
\widehat{\mu}_{11}=3.869 \\
\widehat{\mu}_{12}=4.174 \\
\widehat{\mu}_{13}=4.498
\end{array} \\
& \operatorname{MSE}\left(\widehat{\boldsymbol{\mu}}_{11}^{(t)}\right)=\left(\begin{array}{llll}
0.004 & 0.001 & 0.001 & 0.002 \\
0.001 & 0.004 & 0.003 & 0.001 \\
0.001 & 0.003 & 0.008 & 0.002 \\
0.002 & 0.001 & 0.002 & 0.010
\end{array}\right) \\
& \operatorname{MSE}\left(\widehat{\boldsymbol{\mu}}_{12}^{(t)}\right)=\left(\begin{array}{cccc}
0.004 & 0.001 & 0.001 & 0.002 \\
0.001 & 0.004 & 0.002 & 0.001 \\
0.001 & 0.002 & 0.007 & 0.002 \\
0.002 & 0.001 & 0.002 & 0.009
\end{array}\right)
\end{aligned}
$$




$$
\operatorname{MSE}\left(\widehat{\boldsymbol{\mu}}_{13}^{(t)}\right)=\left(\begin{array}{cccc}
0.004 & 0.001 & 0.001 & 0.001 \\
0.001 & 0.004 & 0.002 & 0.001 \\
0.001 & 0.002 & 0.007 & 0.002 \\
0.001 & 0.001 & 0.002 & 0.0090
\end{array}\right), \quad \begin{aligned}
& M S E\left(\widehat{\mu}_{11}\right)=0.0033 \\
& M S E\left(\widehat{\mu}_{12}\right)=0.0031 \\
& M S E\left(\widehat{\mu}_{13}\right)=0.0029
\end{aligned}
$$

- For $m=30$ and $i=1$

$$
\begin{aligned}
& \widehat{\boldsymbol{\mu}}_{11}^{(t)}=\left(\begin{array}{l}
0.756 \\
1.134 \\
1.484 \\
1.871
\end{array}\right), \quad \widehat{\boldsymbol{\mu}}_{12}^{(t)}=\left(\begin{array}{l}
0.802 \\
1.210 \\
1.601 \\
2.011
\end{array}\right), \quad \widehat{\boldsymbol{\mu}}_{13}^{(t)}=\left(\begin{array}{l}
0.888 \\
1.331 \\
1.747 \\
2.200
\end{array}\right), \quad \begin{array}{l}
\widehat{\mu}_{11}=1.311 \\
\widehat{\mu}_{12}=1.406 \\
\widehat{\mu}_{13}=1.542
\end{array} \\
& \operatorname{MSE}\left(\widehat{\boldsymbol{\mu}}_{11}^{(t)}\right)=10^{-3} \times\left(\begin{array}{cccc}
1.98 & 0.74 & 0.48 & 1.31 \\
0.74 & 1.93 & 1.56 & 0.74 \\
0.48 & 1.56 & 3.73 & 1.22 \\
1.31 & 0.74 & 1.22 & 6.07
\end{array}\right) \\
& \operatorname{MSE}\left(\widehat{\boldsymbol{\mu}}_{12}^{(t)}\right)=10^{-3} \times\left(\begin{array}{cccc}
1.60 & 0.60 & 0.39 & 1.06 \\
0.60 & 5.56 & 1.25 & 0.60 \\
0.39 & 1.25 & 3.00 & 0.99 \\
1.066 & 0.60 & 0.99 & 4.89
\end{array}\right) \\
& \operatorname{MSE}\left(\widehat{\boldsymbol{\mu}}_{13}^{(t)}\right)=10^{-3} \times\left(\begin{array}{cccc}
0.14 & 0.05 & 0.03 & 0.09 \\
0.05 & 0.13 & 0.11 & 0.05 \\
0.03 & 0.11 & 0.26 & 0.08 \\
0.09 & 0.05 & 0.08 & 0.43
\end{array}\right), \quad \begin{array}{l}
M S E\left(\widehat{\mu}_{11}\right)=10^{-3} \times 0.16 \\
M S E\left(\widehat{\mu}_{12}\right)=10^{-3} \times 0.13 \\
M S E\left(\widehat{\mu}_{13}\right)=10^{-3} \times 0.11
\end{array}
\end{aligned}
$$

The results above show that the estimates for parameters of interest are close to the true values and the parametric bootstrap method gives better estimates of MSE when the number of small areas increases.

\section{Concluding remarks}

The demand for small area statistics is for both cross-sectional and for repeated measures data. Small area estimation techniques for repeated measures data under a random effects growth curve model with covariates has been considered in this article. This model accounts for repeated measures data, grouped response units and random effect variations over time. The estimation 
of model parameters has been discussed within a likelihood based approach. We have been able to present the prediction of small area means at each time point and by group units. Finally a parametric bootstrap method for estimating MSE is proposed and a simulation study carried out supports the theoretical results. We thank an anonymous reviewer for constructive suggestion which improved the presentation.

\section{Acknowledgements}

The research of Innocent Ngaruye is supported by the Swedish International Development and Cooperation Agency (SIDA) in collaboration with the University of Rwanda. The research of Dietrich von Rosen is supported by the Swedish Foundation for Humanities and Social Sciences. All involved institutions are acknowledged.

\section{References}

Battese, G. E., Harter, R. M., and Fuller, W. A. (1988). An error-components model for prediction of county crop areas using survey and satellite data. Journal of the American Statistical Association, 83(401):28-36.

Benavent, R. and Morales, D. (2016). Multivariate Fay-Herriot models for small area estimation. Computational Statistics \& Data Analysis, 94:372-390.

Chambers, R. and Clark, R. G. (2012). An introduction to Model-Based Survey Sampling with Applications. Oxford University Press, Oxford, New York.

Consortium, T. (2004). Enhancing small area estimation techniques to meet European needs. Final project report. Office for National Statistics, London.

Datta, G. S., Lahiri, P., Maiti, T., and Lu, K. L. (1999). Hierarchical Bayes estimation of unemployment rates for the states of the US. Journal of the American Statistical Association, 94(448):1074-1082.

Fay, R. E. and Herriot, R. A. (1979). Estimates of income for small places: an application of James-Stein procedures to census data. Journal of the American Statistical Association, 74(366a):269-277.

Filipiak, K. and von Rosen, D. (2012). On MLEs in an extended multivariate linear growth curve model. Metrika, 75(8):1069-1092.

Fujikoshi, Y., Kanda, T., and Ohtaki, M. (1999). Growth curve model with hierarchical withinindividuals design matrices. Annals of the Institute of Statistical Mathematics, 51(4):707-721.

Fuller, W. and Harter, R. (1987). The multivariate components of variance model for small area estimation. Small Area Statistics, pages 103-123. 
Ghosh, M., Datta, G., and Fay, R. (1991). Hierarchical and empirical multivariate bayes analysis in small area estimation. In Proceedings of the Bureau of the Census 1991 Annual Research Conference, pages 63-79, Washington, DC. U.S. Bureau of the Census.

Ghosh, M. and Rao, J. N. K. (1994). Small area estimation: an appraisal. Statistical science, $9(1): 55-76$.

González-Manteiga, W., Lombardía, M., Molina, I., Morales, D., and Santamaría, L. (2008). Bootstrap mean squared error of a small-area EBLUP. Journal of Statistical Computation and Simulation, 78(5):443-462.

Hall, P. and Maiti, T. (2006). On parametric bootstrap methods for small area prediction. Journal of the Royal Statistical Society: Series B, 68(2):221-238.

Harville, D. A. (1998). Matrix Algebra from a Statistician's Perspective. Springer, New York.

Henderson, C. R. (1973). Sire evaluation and genetic trends. Journal of Animal Science, 1973(Symposium):10-41.

Jiongo, V. D., Haziza, D., and Duchesne, P. (2013). Controlling the bias of robust small-area estimators. Biometrika, 100(4):843-858.

Kollo, T. and von Rosen, D. (2005). Advanced Multivariate Statistics with Matrices. Springer, Dordrecht.

Nissinen, K. (2009). Small Area Estimation with Linear Mixed Models from Unit-level panel and Rotating panel data. PhD thesis, University of Jyväskylä.

Nummi, T. (1997). Estimation in a random effects growth curve model. Journal of Applied Statistics, 24(2):157-168.

Pfeffermann, D. (2002). Small area estimation - new developments and directions. International Statistical Review, 70(1):125-143.

Pfeffermann, D. (2013). New important developments in small area estimation. Statistical Science, 28(1):40-68.

Prasad, N. and Rao, J. (1990). The estimation of the mean squared error of small-area estimators. Journal of the American Statistical Association, 85(409):163-171.

Rao, J. N. K. (2003). Small Area Estimation. John Wiley and Sons, New York.

Robinson, G. K. (1991). That BLUP is a good thing: The estimation of random effects. Statistical Science, 6(1):15-32.

Royall, R. M. (1988). The prediction approach to sampling theory. Handbook of Statistics, $6: 399-413$. 
Searle, S. R., Casella, G., and McCulloch, C. E. (2009). Variance Components, volume 391. John Wiley and Sons, New York.

Singh, B. and Sisodia, B. S. V. (2011). Small area estimation in longitudinal surveys. Journal of Reliability and Statistical Studies, 4(2):83-91.

Yokoyama, T. (1995). Statistical inference on some mixed manova-gmanova models with random effects. Hiroshima Mathematical Journal, 25(3):441-474.

Yokoyama, T. and Fujikoshi, Y. (1992). Tests for random-effects covariance structures in the growth curve model with covariates. Hiroshima Mathematical Journal, 22:195-202.

\section{Appendix}

Proof of Lemma 4.1. Obviously, the likelihood equation (7) admits a unique solution for the parameter vector $\boldsymbol{\gamma}$ if the matrix $\boldsymbol{X} \boldsymbol{C}^{\prime o}\left(\boldsymbol{C}^{\prime o}\right)^{\prime} \boldsymbol{X}^{\prime}+\boldsymbol{X} \boldsymbol{R}_{2} \boldsymbol{R}_{2}^{\prime} \boldsymbol{X}^{\prime}$ is of full rank. We have $\operatorname{rank}\left(\boldsymbol{C}^{\prime o}\left(\boldsymbol{C}^{\prime o}\right)^{\prime}\right)=n-m k($ with $n>m k)$ and $\operatorname{rank}\left(\boldsymbol{R}_{2} \boldsymbol{R}_{2}^{\prime}\right)=m k-m$.

Moreover,

$$
\boldsymbol{C}^{\prime o}\left(\boldsymbol{C}^{\prime o}\right)^{\prime} \boldsymbol{R}_{2} \boldsymbol{R}_{2}^{\prime}=\boldsymbol{R}_{2} \boldsymbol{R}_{2}^{\prime} \boldsymbol{C}^{\prime o}\left(\boldsymbol{C}^{\prime o}\right)^{\prime}=\mathbf{0} \text {. So, } \mathcal{C}\left(\boldsymbol{C}^{\prime o}\left(\boldsymbol{C}^{\prime o}\right)^{\prime}\right) \cap \mathcal{C}\left(\boldsymbol{R}_{2} \boldsymbol{R}_{2}^{\prime}\right)=\{\mathbf{0}\}
$$

and then $\operatorname{rank}\left(\boldsymbol{C}^{\prime o}\left(\boldsymbol{C}^{\prime o}\right)^{\prime}+\boldsymbol{R}_{2} \boldsymbol{R}_{2}^{\prime}\right)=\operatorname{rank}\left(\boldsymbol{C}^{\prime o}\left(\boldsymbol{C}^{\prime o}\right)^{\prime}\right)+\operatorname{rank}\left(\boldsymbol{R}_{2} \boldsymbol{R}_{2}^{\prime}\right)=n-m$.

Therefore,

$$
\operatorname{rank}\left(\boldsymbol{X} \boldsymbol{C}^{\prime o}\left(\boldsymbol{C}^{\prime o}\right)^{\prime} \boldsymbol{X}^{\prime}+\boldsymbol{X} \boldsymbol{R}_{2} \boldsymbol{R}_{2}^{\prime} \boldsymbol{X}^{\prime}\right)=\operatorname{rank}\left(\boldsymbol{X}\left(\boldsymbol{C}^{\prime o}\left(\boldsymbol{C}^{\prime o}\right)^{\prime}+\boldsymbol{R}_{2} \boldsymbol{R}_{2}^{\prime}\right) \boldsymbol{X}^{\prime}\right)=\operatorname{rank}(\boldsymbol{X})
$$

provided that $\operatorname{rank}(\boldsymbol{X}) \leq n-m$. The likelihood equation $(8)$ is equivalent to

$$
\boldsymbol{A}^{\prime} \boldsymbol{A B} \boldsymbol{K}_{2} \boldsymbol{K}_{2}^{\prime}=\boldsymbol{A}^{\prime} \boldsymbol{Y} \boldsymbol{R}_{2} \boldsymbol{K}_{2}^{\prime}-\boldsymbol{A}^{\prime} \mathbf{1}_{p} \boldsymbol{\gamma}^{\prime} \boldsymbol{X} \boldsymbol{R}_{2} \boldsymbol{K}_{2}^{\prime} .
$$

This equation in $\boldsymbol{B}$ admits a non unique solution if and only if one or both matrices $\boldsymbol{A}$ and $\boldsymbol{K}_{2}$ are not of full rank. Since $\boldsymbol{A}$ is a full rank matrix, we need to show that the matrix $\boldsymbol{K}_{2}=\boldsymbol{H}\left(\boldsymbol{C} \boldsymbol{C}^{\prime}\right)^{1 / 2} \boldsymbol{\Gamma}_{2}$ is not of full rank. It follows from the construction of the matrix of eigenvectors $\boldsymbol{\Gamma}_{2}$ that $\mathcal{C}\left(\boldsymbol{\Gamma}_{2}\right)=\mathcal{C}\left(\left(\boldsymbol{C} \boldsymbol{C}^{\prime}\right)^{1 / 2}\left(\boldsymbol{C} \boldsymbol{Z}^{\prime}\right)^{o}\right)$. But for any matrices $\boldsymbol{F}$ and $\boldsymbol{G}$ of proper sizes, if $\mathcal{C}(\boldsymbol{F})=\mathcal{C}(\boldsymbol{G})$, then $\mathcal{C}(\boldsymbol{E} \boldsymbol{F})=\mathcal{C}(\boldsymbol{E} \boldsymbol{G})$ (see for example Harville (1998) for more details).

Therefore, $\mathcal{C}\left(\boldsymbol{H}\left(\boldsymbol{C} \boldsymbol{C}^{\prime}\right)^{1 / 2} \boldsymbol{\Gamma}_{2}\right)=\mathcal{C}\left(\boldsymbol{H} \boldsymbol{C} \boldsymbol{C}^{\prime}\left(\boldsymbol{C} \boldsymbol{Z}^{\prime}\right)^{o}\right)$. Moreover, if two matrices have the same column space then they have the same rank. Using this result and the following rank formula which can be found, for example in Kollo and von Rosen (2005), $\operatorname{rank}(\boldsymbol{F}: \boldsymbol{G})=\operatorname{rank}\left(\boldsymbol{F}^{\prime} \boldsymbol{G}^{o}\right)+$ $\operatorname{rank}(\boldsymbol{G})$, we get

$$
\operatorname{rank}\left(\boldsymbol{H}\left(\boldsymbol{C} \boldsymbol{C}^{\prime}\right)^{1 / 2} \boldsymbol{\Gamma}_{2}\right)=\operatorname{rank}\left(\boldsymbol{H} \boldsymbol{C} \boldsymbol{C}^{\prime}\left(\boldsymbol{C} \boldsymbol{Z}^{\prime}\right)^{o}\right)=\operatorname{rank}\left(\boldsymbol{C} \boldsymbol{C}^{\prime} \boldsymbol{H}^{\prime}: \boldsymbol{C Z}^{\prime}\right)-\operatorname{rank}\left(\boldsymbol{C} \boldsymbol{Z}^{\prime}\right) .
$$


Since $\boldsymbol{Z}^{\prime}=\boldsymbol{C}^{\prime} \boldsymbol{Q}$ for

$$
\boldsymbol{Q}=\left(\begin{array}{ccc}
\frac{1}{\sqrt{n_{1}}} \mathbf{1}_{k} & & \mathbf{0} \\
& \ddots & \\
\mathbf{0} & & \frac{1}{\sqrt{n_{m}}} \mathbf{1}_{k}
\end{array}\right)
$$

it follows that

$$
\begin{aligned}
\operatorname{rank}\left(\boldsymbol{H}\left(\boldsymbol{C C}^{\prime}\right)^{1 / 2} \boldsymbol{\Gamma}_{2}\right) & =\operatorname{rank}\left(\boldsymbol{H} \boldsymbol{C}^{\prime}\left(\boldsymbol{C} \boldsymbol{Z}^{\prime}\right)^{o}\right)=\operatorname{rank}\left(\boldsymbol{C}^{\prime} \boldsymbol{H}^{\prime}: \boldsymbol{C C}^{\prime} \boldsymbol{Q}\right)-\operatorname{rank}\left(\boldsymbol{C} \boldsymbol{C}^{\prime} \boldsymbol{Q}\right) \\
& =\operatorname{rank}\left(\boldsymbol{H}^{\prime}: \boldsymbol{Q}\right)-\operatorname{rank}(\boldsymbol{Q}) \\
& =\operatorname{rank}\left(\boldsymbol{H}^{\prime}\right)+\operatorname{rank}(\boldsymbol{Q})-\operatorname{dim}\left(\mathcal{C}\left(\boldsymbol{H}^{\prime}\right) \cap \mathcal{C}(\boldsymbol{Q})\right)-\operatorname{rank}(\boldsymbol{Q}) \\
& =\operatorname{rank}\left(\boldsymbol{H}^{\prime}\right)-\operatorname{dim}\left(\mathcal{C}\left(\boldsymbol{H}^{\prime}\right) \cap \mathcal{C}(\boldsymbol{Q})\right),
\end{aligned}
$$

since the matrix $\boldsymbol{C} \boldsymbol{C}^{\prime}$ is of full rank and where dim denotes the dimension of a subspace. It remains to show that $\mathcal{C}\left(\boldsymbol{H}^{\prime}\right)$ and $\mathcal{C}(\boldsymbol{Q})$ are not disjoint.

Let $\boldsymbol{v}_{1}=\mathbf{1}_{k}$ and $\boldsymbol{v}_{2}=\left(\sqrt{n_{1}}, \cdots, \sqrt{n_{m}}\right)^{\prime}$ and that $\boldsymbol{H}=\left(\boldsymbol{I}_{k}: \cdots: \boldsymbol{I}_{k}\right)$. Then we have $\boldsymbol{H}^{\prime} \boldsymbol{v}_{1}=\mathbf{1}_{m k}$ and $\boldsymbol{Q} \boldsymbol{v}_{2}=\mathbf{1}_{m k}$. Thus, the two spaces are not disjoint since they include a common vector. This completes the proof of the lemma. 